\title{
Textured Contact Lens based Iris Presentation Attack in Uncontrolled Environment
}

\author{
Daksha Yadav \\ West Virginia University, dayadav@mix.wvu.edu
}

Follow this and additional works at: https://researchrepository.wvu.edu/etd

Part of the Bioimaging and Biomedical Optics Commons, and the Computer Engineering Commons

\section{Recommended Citation}

Yadav, Daksha, "Textured Contact Lens based Iris Presentation Attack in Uncontrolled Environment" (2019). Graduate Theses, Dissertations, and Problem Reports. 3940.

https://researchrepository.wvu.edu/etd/3940

This Thesis is protected by copyright and/or related rights. It has been brought to you by the The Research Repository @ WVU with permission from the rights-holder(s). You are free to use this Thesis in any way that is permitted by the copyright and related rights legislation that applies to your use. For other uses you must obtain permission from the rights-holder(s) directly, unless additional rights are indicated by a Creative Commons license in the record and/ or on the work itself. This Thesis has been accepted for inclusion in WVU Graduate Theses, Dissertations, and Problem Reports collection by an authorized administrator of The Research Repository @ WVU. For more information, please contact researchrepository@mail.wvu.edu. 


\section{Textured Contact Lens based Iris Presentation Attack in Uncontrolled Environment}

\section{Daksha Yadav}

Thesis submitted to the

Benjamin M. Staler College of Engineering and Mineral Resources at West Virginia University

in partial fulfillment of the requirements for the degree of

Master of Science

in

Computer Science

Afzel Noore, Ph.D., Chair

Mayank Vatsa, Ph.D.

Richa Singh, Ph.D.

Lane Department of Computer Science and Electrical Engineering

Morgantown, West Virginia

2018

Keywords: Iris Recognition, Presentation Attack, Textured Contact Lens

Copyright 2018 Daksha Yadav 


\begin{abstract}
Textured Contact Lens Based Iris Presentation Attack In Uncontrolled Environment Daksha Yadav
\end{abstract}

The widespread use of smartphones has spurred the research in mobile iris devices. Due to their convenience, these mobile devices are also utilized in unconstrained outdoor conditions. At the same time, iris recognition in the visible spectrum has developed into an active area of research. These scenarios have necessitated the development of reliable iris recognition algorithms for such an uncontrolled environment. Additionally, iris presentation attacks such as textured contact lens pose a major challenge to current iris recognition systems.

Motivated by these factors, in this thesis, a detailed analysis of the effect of textured contact lenses on iris recognition in an uncontrolled environment is presented by creating Mobile Uncontrolled Iris Presentation Attack Database. It consists of more than 10,000 iris images of subjects wearing textured contact lens and without wearing contact lenses captured in an indoor and outdoor environment using a mobile iris sensor. The first contact lens database in the visible spectrum is also introduced, named as UVCLI database. Using this database, a detailed analysis of the effect of textured contact lenses on iris recognition in the visible spectrum is presented. It is observed that textured contact lenses degrade the unconstrained iris recognition performance by over $25 \%$ and thus, may be utilized intentionally or unintentionally to attack existing iris recognition systems. Also, the first study to investigate the impact of textured contact lenses on identity impersonation is performed. The results demonstrate that a perpetrator can impersonate an enrolled subject by wearing the same textured contact lens.

This thesis also presents a novel algorithm, DensePAD, which utilizes DenseNet based convolutional neural network architecture for the near-infrared spectrum based iris presentation attack detection. The proposed algorithm is trained on a combined iris database of more than 270, 000 real and attack iris images. In-depth experimental evaluation of this algorithm reveals its superior performance in detecting iris presentation attack images on the combined iris database as well as the proposed MUIPAD. 
This thesis is dedicated to my parents, who have always encouraged me to pursue my dreams, especially this one ... 


\section{Acknowledgements}

I wish to utilize this opportunity to thank all the people who in one way or another contributed to the completion of this thesis.

First and foremost, I gratefully acknowledge my advisor Dr. Afzel Noore for his continued guidance and motivation throughout this journey. His wise words motivated me to remain patient even during tough times and challenged me to think about novel solutions. I am also highly thankful to my sources of inspiration, Dr. Richa Singh and Dr. Mayank Vatsa for guiding me since my undergraduate degree. Their hard work, perseverance, and curiosity to work on new research problems provoked me to choose the path of research.

Finally, I am highly grateful to my parents for making immeasurable sacrifices for me. They have been my constant pillars of support and I will continue to strive every day to make them proud. I express gratitude to my brother, Adit and sister-in-law, Poonam for supporting my decisions and being my towers of strength. Thank you to all of you! 


\section{Dissemination of Research Results}

- D. Yadav, N. Kohli, M. Vatsa, R. Singh, and A. Noore. "Iris presentation attack detection in uncontrolled environment using DenseNet." Under Review.

- D. Yadav, N. Kohli, S. Yadav, M. Vatsa, R. Singh, and A. Noore. "Iris presentation attack via textured contact lens in unconstrained environment." In IEEE Winter Conference on Applications of Computer Vision, pp. 503-511, 2018.

- D. Yadav, N. Kohli, A. Akshay, M. Vatsa, R. Singh, and A. Noore. "Fusion of handcrafted and deep learning features for large-scale multiple iris presentation attack detection." In IEEE Conference on Computer Vision and Pattern Recognition Workshops, pp. 572-579, 2018.

- D. Yadav, N. Kohli, M. Vatsa, R. Singh, and A. Noore. "Unconstrained visible spectrum iris with textured contact lens variations: Database and benchmarking." In IEEE International Joint Conference on Biometrics, pp. 574-580, 2017. 


\section{Table of contents}

Dedication iii

Acknowledgements $\quad$ iv

Dissemination of Research Results v v

List of figures $\quad$ ix

List of tables $\quad$ xi

Nomenclature $\quad$ xiii

1 Introduction 1

1.1 Iris based Biometric Authentication . . . . . . . . . . . . . . 1

1.2 Motivation and Literature Review _. . . . . . . . . . . . . 3

1.3 Contributions of the Thesis . . . . . . . . . . . . . 5

2 Proposed Databases 9

2.1 Mobile Uncontrolled Iris Presentation Attack Database (MUIPAD) . . . . . 9

2.1 .1 Database Characteristics ................. 11

2.2 Unconstrained Visible Spectrum Contact Lens Iris

(UVCLI) Database . . . . . . . . . . . . . . . . . . . . 12 
2.2.1 Database Characteristics . . . . . . . . . . . . . . 12

3 Influence of Uncontrolled Environment and Textured Contact Lens on Iris Recog$\begin{array}{ll}\text { nition } & 16\end{array}$

3.1 Effect of Uncontrolled Environment on NIR Iris Recognition . . . . . . . 17

$3.1 .1 \quad$ Experimental Protocol . . . . . . . . . . . . . . . . 17

3.1.2 Experimental Results . . . . . . . . . . . . . . . . . 18

3.2 Effect of Textured Contact Lens on NIR Iris Recognition . . . . . . . . . . 20

3.2.1 Experimental Protocol . . . . . . . . . . . . . . . 20

3.2.2 Experimental Results . . . . . . . . . . . . . . . . 21

3.3 Effect on Visible Spectrum Iris Recognition . . . . . . . . . . . . . 21

3.3.1 Iris Recognition Algorithms for Evaluation . . . . . . . . . . . 21

$3.3 .2 \quad$ Experimental Protocol $\ldots \ldots . \ldots 22$

3.3.3 Experimental Results . . . . . . . . . . . . . . . . . . . 24

3.4 Textured Contact Lens for Identity Impersonation and Obfuscation . . . . . 26

3.4.1 Experimental Protocol . . . . . . . . . . . . . . . 26

3.4.2 Experimental Results . . . . . . . . . . . . . . . . . 28

4 Benchmarking Existing Textured Contact Lens Detection Algorithms 30

4.1 Performance on Mobile Uncontrolled Iris Presentation Attack Database . . 30

4.1.1 Benchmarking PAD performance . . . . . . . . . 32

4.1.2 Evaluating the Effect of Unseen Environment . . . . . . . . . . . 33

4.1.3 Examining the Impact of Unseen Textured Contact Lens Manufacturer 35

4.2 Performance on Unconstrained Visible Spectrum Contact Lens Iris Database 36

$4.2 .1 \quad$ Experimental Setup . . . . . . . . . . . . . . 36

4.2.2 Experimental Results . . . . . . . . . . . . . . . . 37 
5 Proposed DenseNet based Algorithm for Iris Presentation Attack Detection

5.1 Proposed DensePAD Algorithm for Iris Presentation Attack Detection . . . 42

5.2 Experimental Evaluation $\ldots \ldots \ldots \ldots 4 . \ldots \ldots$

5.2.1 Combined Iris Database $\ldots \ldots . \ldots . \ldots 44$

5.2 .2 Experimental Setup . . . . . . . . . . . . . . . . . 47

5.2.3 Results on Combined Iris Database . . . . . . . . . . . . . . 47

5.2.4 Results on Proposed MUIPAD . . . . . . . . . . . . . . . 48

6 Conclusion and Future Work $\quad 50$

$\begin{array}{ll}\text { Bibliography } & 53\end{array}$ 


\section{List of figures}

1.1 Illustrating the different regions of the human eye. . . . . . . . . . . 2

1.2 Illustrating the variations due to textured contact lenses and environmental variations in near-infrared and visible spectrum iris images. (Best viewed in

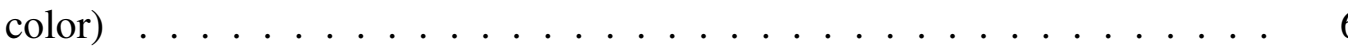

2.1 Showcasing the variations in iris images due to textured contact lens and unconstrained environmental conditions. Figure (a): real iris images captured indoor, (b): real iris images captured outdoor, (c): textured contact lens iris images captured indoor, and (d): textured contact lens iris images captured outdoor. .......................... 10

2.2 Sample iris images of a subject from the proposed Mobile Uncontrolled Iris Presentation Attack Database. $\ldots . . \cdots \cdots$

2.3 Sample images of a subject from the proposed Unconstrained Visible Contact Lens Iris (UVCLI) database demonstrating the variations due to textured contact lens, environmental conditions, and multi-session acquisition. (Best viewed in color $) \ldots \ldots \ldots \ldots$. . . . . . . . . . . . 14 
3.1 ROC curves illustrating the influence of uncontrolled environment and textured contact lens on the performance of iris recognition systems on the combined session. . . . . . . . . . . . . . . . . . . . 19

3.2 Sample segmentation outputs of visible iris images from the proposed UVCLI database using OSIRIS V4.1 [37] (row 1) and TV based segmentation algorithm [44] (row 2). (Best viewed in color) . . . . . . . . . . . . . .

3.3 ROC curves showing the performance of visible iris recognition on the proposed UVCLI database (Session 1 and Session 2 combined).

3.4 Illustrating sample instances of identity impersonation and identity obfuscation using textured contact lenses.

4.1 ROC curves showing the performance of visible spectrum iris PAD algorithms on the proposed UVCLI database. . . . . . . . . . . . . 38

5.1 Architecture of proposed DensePAD algorithm for textured contact lens

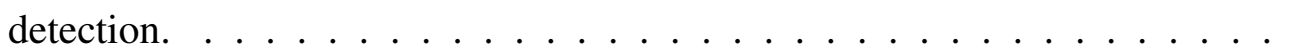

5.2 Sample iris image types from various constituent databases of the Combined Iris database $\ldots \ldots \ldots \ldots \ldots \ldots$ 


\section{List of tables}

1.1 Information about number of subjects, number of images, and the iris image acquisition devices in the existing iris presentation attack databases since 2010. 4

1.2 Characteristics (type of presentation attacks and acquisition environment) of the existing iris presentation attack databases. . . . . . . . . . . 5

2.1 Characteristics of the proposed Unconstrained Visible Contact Lens Iris (UVCLI) database. . . . . . . . . . . . . . . . . . 12

3.1 Performance (Equal Error Rate \%) of iris recognition systems in the presence of uncontrolled environment and textured contact lens using (a) VeriEye COTS iris recognition system and (b) OSIRIS iris recognition system. . . . 18

3.2 Iris recognition performance (EER\%) on the proposed UVCLI database. . . 24

3.3 Percentage of scores labeled as genuine by VeriEye for different scenarios of identity impersonation and identity obfuscation. . . . . . . . . . . 28

4.1 Iris presentation attack detection performance on the proposed Mobile Uncontrolled Iris Presentation Attack Database (MUIPAD). The error values are reported in $\% \ldots \ldots \ldots \ldots \ldots \ldots$ 
4.2 Iris presentation attack detection performance (in \%) of AlexNet [24] on iris images acquired in unseen environment with textured contact lens. In the Benchmark experiment, training and testing partitions contain real and textured lens iris images captured indoors and outdoors across different sessions. .......................... 33

4.3 Iris presentation attack detection performance (in \%) of AlexNet [24] to showcase the effect of unseen textured contact lens manufacturer. . . . . . . 34

4.4 Iris Presentation Attack Detection Performance on the proposed UVCLI database. . . . . . . . . . . . . . . . . . .

5.1 Characteristics of the Combined Iris Database and its constituent databases. 44

5.2 The number of real and attacked iris images in each fold for the Combined Iris database. . . . . . . . . . . . . . . . . . . . . . . .

5.3 Iris presentation attack detection performance (\%) of the proposed DensePAD and existing algorithms on the Combined Iris database. $\ldots \ldots \ldots$

5.4 Iris presentation attack detection performance of the proposed DensePAD algorithm on the proposed Mobile Uncontrolled Iris Presentation Attack Database (MUIPAD). The error values are reported in \% . . . . . . . . . . 48 


\title{
Nomenclature
}

\author{
Acronyms / Abbreviations
}

APCER Attack Presentation Classification Error Rate

BPCER Bonafide Presentation Classification Error Rate

COTS Commercial-Off-The-Shelf

EER Equal Error Rate

MUIPAD Mobile Uncontrolled Iris Presentation Attack Database

NIR Near Infrared

PAD Presentation Attack Detection

ROC Receiver Operating Characteristic

TV Total Variation

UVCLI Unconstrained Visible Spectrum Contact Lens Iris Database 


\section{Chapter 1}

\section{Introduction}

\subsection{Iris based Biometric Authentication}

Biometrics refers to the use of physical or behavioral traits (such as the face, iris, fingerprints, and voice) for the purpose of establishing one's unique identity [19]. A good biometric trait exhibits traits such as universality, distinctiveness, permanence, and collectability. Iris is one of the most reliable and accurate biometric modalities due to the highly unique property of iris tissue structure. The iris tissue has rich textural information and is unique for each individual as well as differs between two eyes of the same individual. Figure 1.1 illustrates the different regions of an eye: sclera, iris, and pupil.

Daugman patented the first successful iris recognition algorithm [7]. His algorithm was based on a test of statistical independence of the phase of Gabor wavelets fitted on a grid of locations superimposed on a pseudo-polar transformation of the iris texture. That basic design still remains the dominant iris recognition method. Traditional iris recognition systems operate in the near-infrared (NIR) spectrum. During the iris image acquisition, the light in the NIR spectrum permeates the surface of the eye and highlights the unique iris 


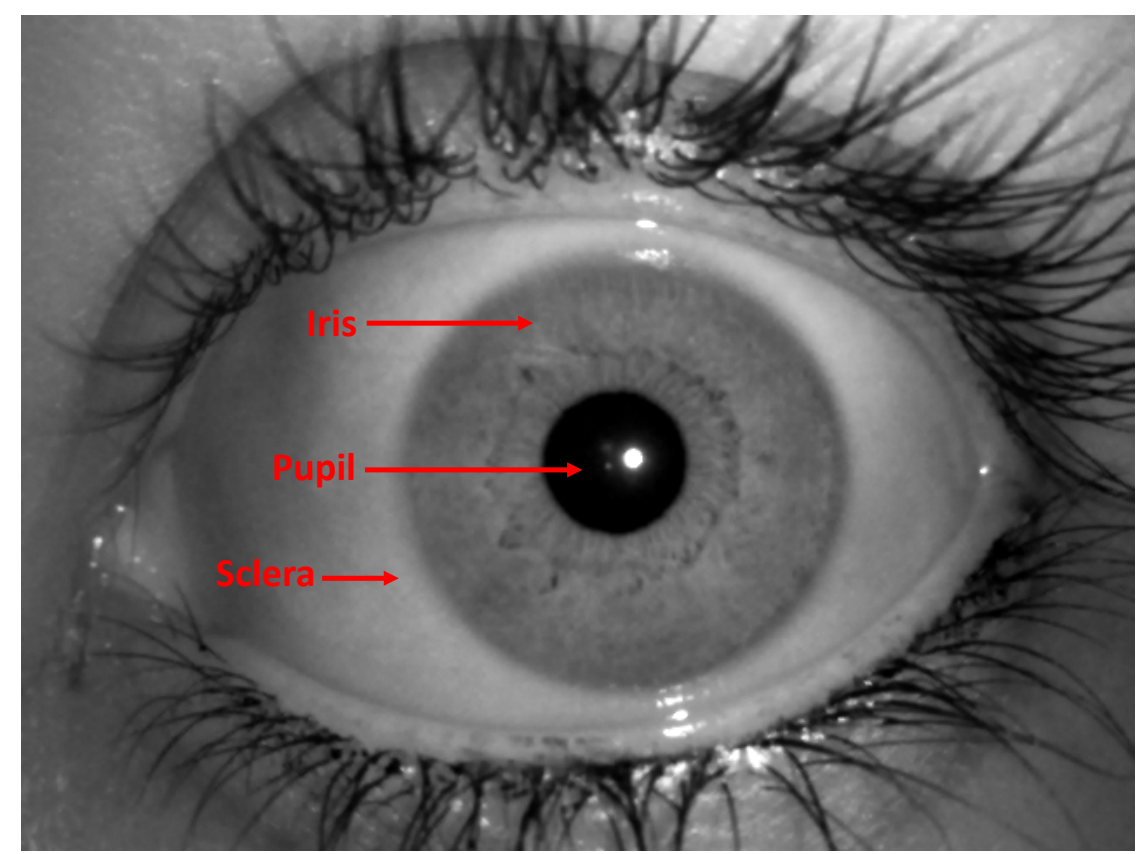

Figure 1.1: Illustrating the different regions of the human eye.

texture patterns. Due to its accuracy, iris recognition has been used successfully in numerous applications including large-scale national identification projects and border security.

It is estimated that over 2.71 billion individuals are expected to own a smartphone by the year 2019 [1]. This staggering growth of smartphones has contributed to the emerging field of mobile biometrics with the increasing number of small factor biometric sensors. Apart from the robust nature of traditional biometrics, mobile biometrics offer portability as a key advantage [20]. The mobile nature of these sensors facilitates their deployment in a variety of applications such as e-banking and authentication for e-commerce applications.

Research in visible spectrum iris recognition $[18,32,38]$ has witnessed significant growth in recent years and is being actively explored. Iris recognition in the visible spectrum can be utilized for applications such as authentication in outdoor environments where near-infrared iris images may be difficult to acquire. Visible spectrum iris recognition has also gained popularity due to its potential application in the field of mobile biometrics [35, 39]. 


\subsection{Motivation and Literature Review}

Due to the reliable nature of iris biometrics [28], iris sensors and recognition systems are being made available in the new generation mobile smartphones [31]. This feature is proving advantageous in many scenarios but has also introduced unforeseen research challenges. For instance, acquisition of iris images may be challenging in outdoor locations during daytime and in high illumination settings. Other challenges such as complexity of algorithms also need to be considered in their deployment. However, the majority of the research is focusing on the controlled environment and existing databases contain iris images captured using traditional close-capture iris devices.

The surge of iris biometrics in authentication applications such as banking and ecommerce sectors has increased concerns about the ease of subversion of these systems by determined adversaries. In the literature, researchers have demonstrated the impact of a variety of presentation attacks on the near-infrared spectrum based iris recognition systems such as print/scan attacks [15], textured contact lens [21], and synthetic irises [36]. Studies have also focused on developing algorithms for detection of contact lenses in iris images captured in NIR spectrum [9, 23, 40] (as shown in Tables 1.1 and 1.2). In 2010, Baker et al. [3] demonstrated that transparent/clear contact lenses with no visible texture cause a small degradation in iris recognition performance. Later, Doyle et al. [9] and Kohli et al. [21] showed that wearing textured/cosmetic contact lenses nearly guarantees a false non-match output. Yadav et al. [40] created IIIT-Delhi Contact Lens Iris database and observed 22\%$38 \%$ reduction in iris verification performance when matching an image of an iris with no contact lens with an iris image with a textured contact lens. Doyle and Bowyer [8] developed ND-Contact-Lens-2015 database and proposed using binarized statistical image features for detecting textured contact lenses in a given iris image. Apart from these databases, LivDet 
Table 1.1: Information about number of subjects, number of images, and the iris image acquisition devices in the existing iris presentation attack databases since 2010.

\begin{tabular}{|l|c|c|l|}
\hline Database & No. of Subjects & No. of Images & Acquisition Device \\
\hline $\begin{array}{l}\text { ND-Iris-Contact-Lens- } \\
2010 \\
{[3]}\end{array}$ & 211 & 21,700 & LG2200 \\
\hline $\begin{array}{l}\text { ND-Contact-Lens- } \\
2015 \\
{[8]}\end{array}$ & 326 & 7,300 & LG4000, AD100 \\
\hline $\begin{array}{l}\text { IIIT-Delhi Contact Lens } \\
\text { Iris Database [40] }\end{array}$ & 101 & 6,570 & CIS 202 \\
\hline $\begin{array}{l}\text { IIIT-Delhi Iris Spoofing } \\
\text { Database [15] }\end{array}$ & 101 & 4,848 & $\begin{array}{l}\text { HP Color LaserJet 2025 } \\
\text { \& CIS 202 (Iris Sensor) }\end{array}$ \\
\hline $\begin{array}{l}\text { ATVS-FIr [11] } \\
\text { LivDet-Iris-2013- }\end{array}$ & 50 & 1,600 & $\begin{array}{l}\text { LG Iris Access } \\
\text { EOU3000 }\end{array}$ \\
\hline $\begin{array}{l}\text { Warsaw } \\
\text { [41] }\end{array}$ & 284 & 1,667 & $\begin{array}{l}\text { IrisGuard AD100, HP } \\
\text { LaserJet 1320, Lexmark } \\
\text { c534dn }\end{array}$ \\
\hline $\begin{array}{l}\text { LivDet-Iris-2015- } \\
\text { Clarkson } \\
\text { [42] }\end{array}$ & 45 & 3,726 & $\begin{array}{l}\text { LG IrisAccess } \\
\text { EOU2200 }\end{array}$ \\
\hline
\end{tabular}

(liveliness detection) challenges $[41,42]$ have been conducted in the research community to demonstrate recent challenges in texture contact lens detection.

Similar to NIR spectrum, various visible spectrum iris databases exist in the literature such as UBIRIS.v2 [32], MICHE [26], mobile phone-based [39], and VSSIRIS [35]. However, there is a distinct lack of attention towards the probability of presentation attacks in visible spectrum based iris recognition and very few relevant studies exist. Recently, Raghavendra and Busch [33] have demonstrated a video-replay presentation attack for iris recognition in the visible spectrum using printed photos and electronic displays. Even though the textured contact lens is becoming more popular with developments in low-cost technology, their influence on iris recognition in visible spectrum has not been explored. The problem is compounded by the non-existence of any visible spectrum iris database with annotated 
Table 1.2: Characteristics (type of presentation attacks and acquisition environment) of the existing iris presentation attack databases.

\begin{tabular}{|l|c|c|c|c|}
\hline Database & $\begin{array}{c}\text { Textured } \\
\text { Contact Lens }\end{array}$ & $\begin{array}{c}\text { Print } \\
\text { Attack }\end{array}$ & $\begin{array}{c}\text { Uncontrolled } \\
\text { Environment }\end{array}$ & $\begin{array}{c}\text { Mobile } \\
\text { Sensor }\end{array}$ \\
\hline $\begin{array}{l}\text { ND-Iris-Contact-Lens- } \\
\text { 2010[3] }\end{array}$ & $\checkmark$ & $x$ & $x$ & $x$ \\
\hline $\begin{array}{l}\text { ND-Contact-Lens- } \\
2015[8]\end{array}$ & $\checkmark$ & $x$ & $x$ & $x$ \\
\hline $\begin{array}{l}\text { IIIT-Delhi Contact Lens } \\
\text { Iris Database[40] }\end{array}$ & $\checkmark$ & $x$ & $x$ & $x$ \\
\hline $\begin{array}{l}\text { IIIT-Delhi Iris Spoofing } \\
\text { Database[15] }\end{array}$ & $x$ & $\checkmark$ & $x$ & $x$ \\
\hline ATVS-FIr [11] & $x$ & $\checkmark$ & $x$ & $x$ \\
\hline $\begin{array}{l}\text { LivDet-Iris-2013- } \\
\text { Warsaw[41] }\end{array}$ & $\checkmark$ & $\checkmark$ & $x$ & $x$ \\
\hline $\begin{array}{l}\text { LivDet-Iris-2015- } \\
\text { Clarkson[42] }\end{array}$ & $\checkmark$ & $\checkmark$ & $x$ & $x$ \\
\hline
\end{tabular}

information regarding subjects wearing textured contact lens. Moreover, it is imperative to evaluate the effectiveness of these algorithms in detecting contact lenses in iris images captured in unconstrained scenarios. Figure 1.2 highlights the transformations in the iris texture patterns due to textured contact lenses and environmental variations in NIR and visible spectrum.

\subsection{Contributions of the Thesis}

Presentation attacks are performed with the goal of obfuscating the true identity of the attacker or impersonating a specific person. By obfuscation, one can achieve multiple enrollments to avail facilities that utilize biometric recognition. Through impersonation, one can gain access to the specific facilities available to a specific user. However, as shown in Table 1.2, none of the existing databases have focused on (i) textured contact lenses, (ii) print attack, 

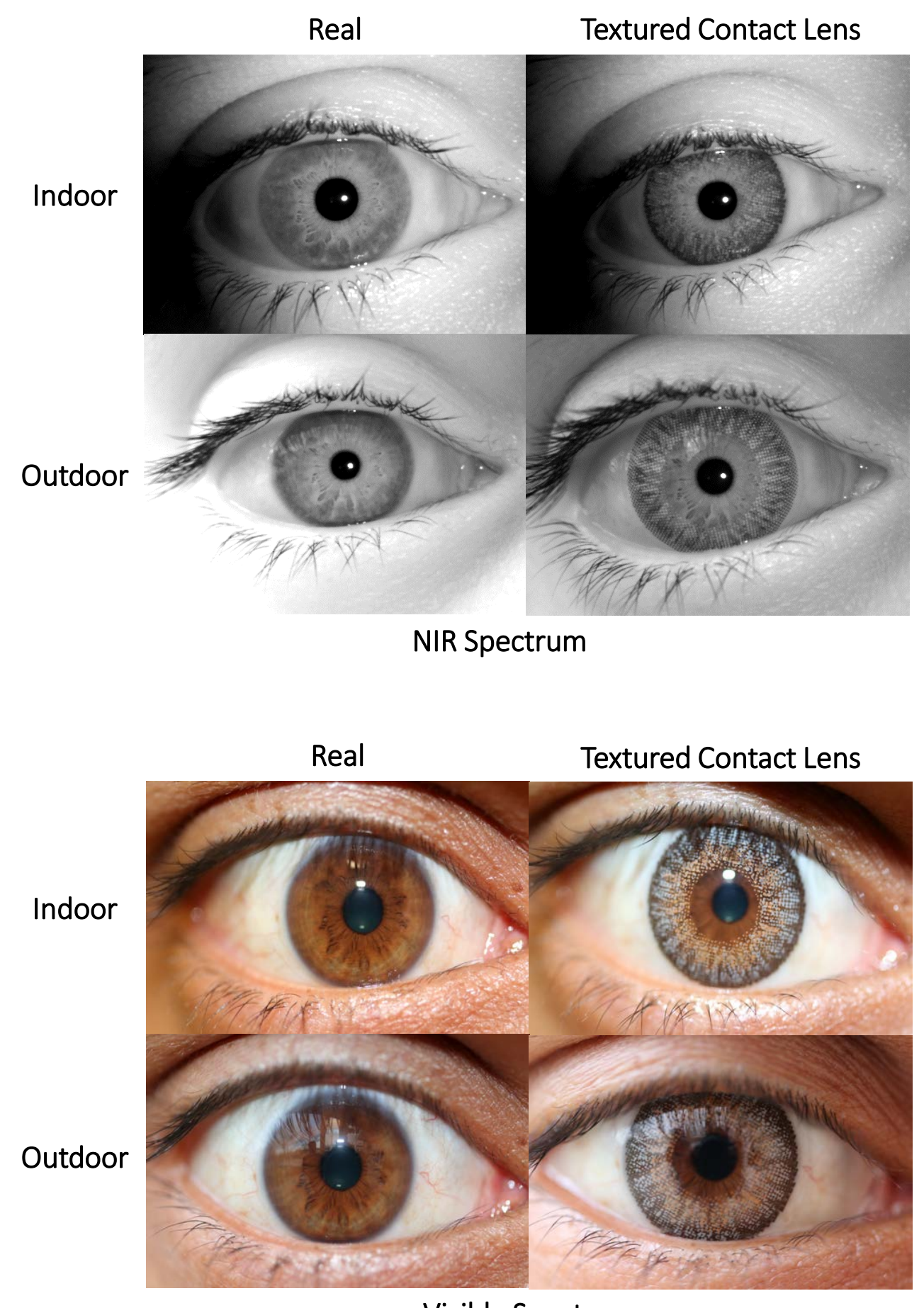

Visible Spectrum

Figure 1.2: Illustrating the variations due to textured contact lenses and environmental variations in near-infrared and visible spectrum iris images. (Best viewed in color) 
(iii) uncontrolled environment, and (iv) data captured using a mobile device, simultaneously. This research attempts to fill this gap by:

- introducing a novel database named as Mobile Uncontrolled Iris Presentation Attack Database (MUIPAD). This database has with and without textured contact lens iris images acquired in uncontrolled environmental variations. The images in the proposed database have been acquired using a low form factor mobile iris scanner. This database contains over 10,000 multi-session iris images, belonging to 70 eye classes. The database includes corresponding printed iris images generated using two printers to simulate the print attack. This is the first publicly available iris database offering a unique combination of such variations.

- developing a new benchmark unconstrained visible spectrum contact lens iris database termed as Unconstrained Visible Spectrum Contact Lens Iris (UVCLI) Database. The proposed database consists of over 3,800 images, both real and with textured contact lenses, pertaining to 70 iris classes. This database comprises multi-session images of subjects captured in a controlled indoor environment as well as in an unconstrained outdoor environment with variations in illumination. To the best of our knowledge, this is the first such publicly available visible spectrum database. Since the database contains multiple images per class, it can also be utilized to create better iris recognition algorithms in the visible spectrum.

- evaluating the effect of textured contact lenses on the performance of the existing visible spectrum iris recognition algorithm using the proposed UVCLI database. The influence of environmental variations at the time of image acquisition is also analyzed. Therefore, the influence of two confounding variables: textured contact lenses and environmental variations is examined. 
- presenting an in-depth analysis of the efficacy of textured contact lenses in accomplishing identity impersonation and identity obfuscation. To the best of our knowledge, this is the first study to examine if textured contact lenses can be utilized by adversaries for both identity impersonation as well as obfuscation.

- demonstrating the performance of deep learning based features as well as existing iris presentation attack detection techniques on the proposed MUIPAD database. The impact of unknown environmental conditions and unknown contact lens manufacturer on presentation attack detection is evaluated to simulate real-world scenarios. The performance of existing iris presentation attack detection algorithms on the proposed UVCLI database is benchmarked in detecting contact lenses in the visible spectrum iris images.

- presenting a novel algorithm, DensePAD, which utilizes DenseNet based convolutional neural network architecture for near-infrared spectrum based iris presentation attack detection. The proposed algorithm is trained a combined iris database of more than 270, 000 real and attack iris images. 


\section{Chapter 2}

\section{Proposed Databases}

The portable nature of mobile iris based systems enables their usage in outdoor scenarios. However, acquiring iris images in uncontrolled settings may deteriorate the performance of iris recognition systems. There is a need to develop databases which encompass iris images acquired in unconstrained settings to facilitate the research in mobile iris recognition. Further, the advent in cutting-edge technology has led to consumers being offered textured contact lenses with diverse choices of colors and textures by different manufacturers. This illustrates an urgent need to analyze if textured contact lenses can be utilized with a malicious intent to impersonate or obfuscate one's identity. Figure 2.1 illustrates the variations in iris occurring due to the presence of textured contact lenses and uncontrolled environment.

\subsection{Mobile Uncontrolled Iris Presentation Attack Database (MUIPAD)}

There is no database available to the research community which provides a combination of all the aforementioned variations. This thesis presents the Mobile Uncontrolled Iris Presentation 


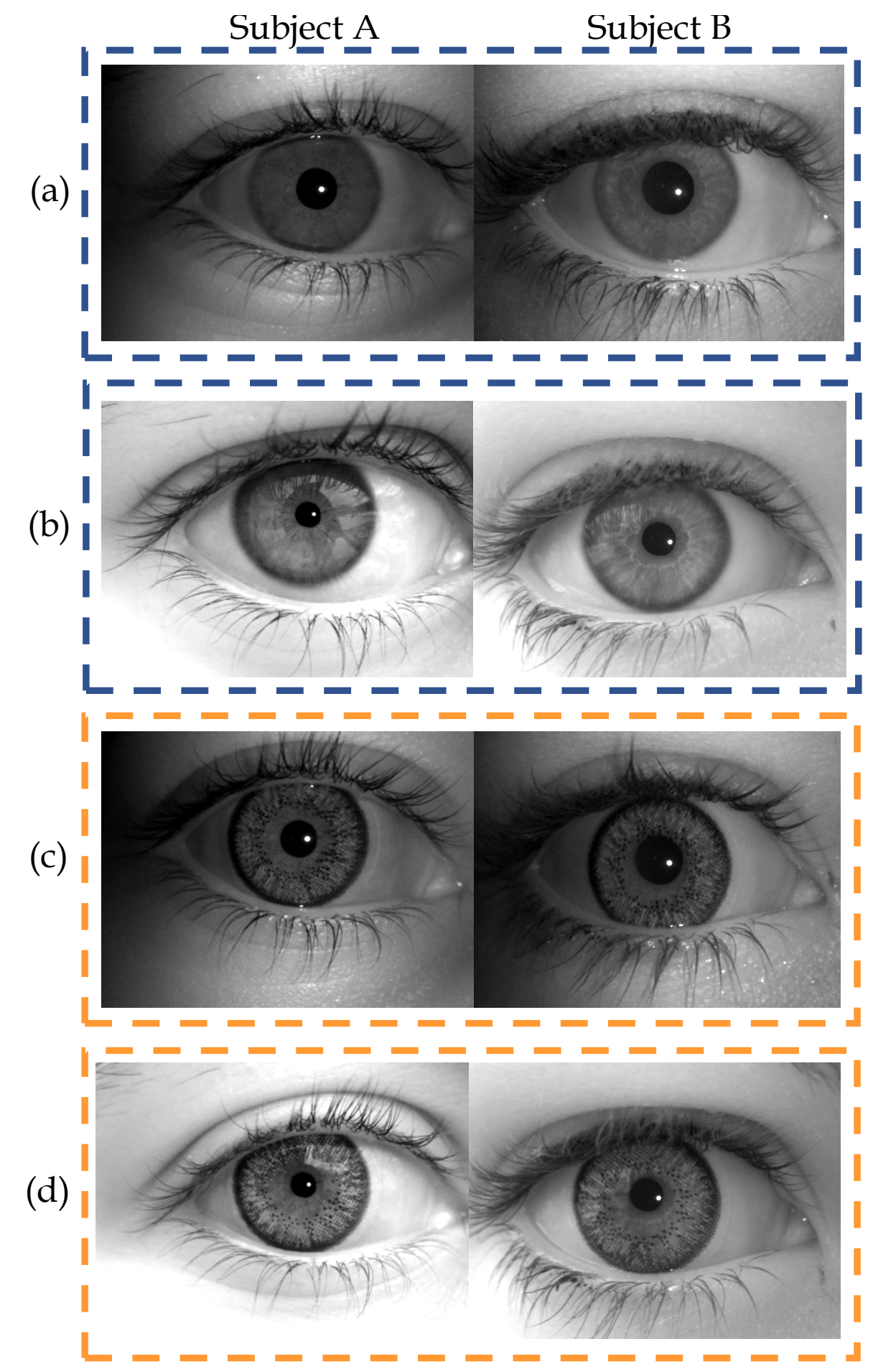

Figure 2.1: Showcasing the variations in iris images due to textured contact lens and unconstrained environmental conditions. Figure (a): real iris images captured indoor, (b): real iris images captured outdoor, (c): textured contact lens iris images captured indoor, and (d): textured contact lens iris images captured outdoor. 
Attack Database (MUIPAD) to analyze the effect of uncontrolled environmental and textured contact lenses on iris images captured using a mobile sensor. The database will be available to the research community at http://iab-rubric.org/resources.html\#iris to promote research in this area.

\subsubsection{Database Characteristics}

The iris images in the proposed MUIPAD database have been acquired using the IriShield MK2120U mobile sensor. For each subject in the database, images have been captured indoors (controlled environment) and outdoors (uncontrolled environment) in a multi-session protocol. In each session, with and without textured contact lens iris images of the subjects are captured indoors as well as outdoors. It is also ensured that subjects are provided with different textured contact lenses for the two sessions. It should be noted that the outdoor images have been captured in varying time of the day and weather conditions. A minimum of five images of each eye is acquired during each session. The time gap between the two sessions is at least one day.

The textured contact lenses utilized in the proposed databases have been grouped into the following categories based on the manufacturer: Freshlook Dailies (one-day disposable lens), Freshlook Colorblends (weekly disposable lens), Bausch + Lomb Lacelle (monthly disposable), Aryan 3-tone (yearly disposable), and Celebration (weekly disposable lens).

Additionally, print attack images have also been included in the proposed database. All iris images (with and without textured contact lens) are printed using HP LaserJet Enterprise P3015 (black and white) and Konica Minolta Bizhub C454E (colored). This is followed by scanning of the printed images using Konica Minolta Bizhub C454E scanner. Thus, the proposed database comprises 10,296 iris images from 35 subjects (18 males and 17 females) with 70 eye classes. The subjects in the database belong to different ethnicities such as 
Table 2.1: Characteristics of the proposed Unconstrained Visible Contact Lens Iris (UVCLI) database.

\begin{tabular}{|l|l|}
\hline Spectrum & Visible \\
\hline Environmental Variations & $\begin{array}{l}\text { Multi-Session Acquisition Indoors and } \\
\text { Outdoors }\end{array}$ \\
\hline Total Number of Images & 3,802 \\
\hline Types of Images & Real $(1,877)$ \& Textured Contact Lens $(1,925)$ \\
\hline Contact Lens Brands & $\begin{array}{l}\text { CIBA Vision Freshlook Colorblends; CIBA } \\
\text { Vision Dailies; Bausch \& Lomb Lacelle; Aryan; } \\
\text { and Celebration }\end{array}$ \\
\hline Number of Subjects (Classes) & $35(70)$ \\
\hline Participant Gender & Females (17) \& Males (18) \\
\hline
\end{tabular}

Caucasian and Asian. Figure 2.2 illustrates sample real, textured contact lens, and printed iris images captured in uncontrolled environmental scenarios from the proposed database.

\subsection{Unconstrained Visible Spectrum Contact Lens Iris}

\section{(UVCLI) Database}

\subsubsection{Database Characteristics}

This thesis also presents the Unconstrained Visible Spectrum Contact Lens Iris (UVCLI) database. The proposed UVCLI database consists of 3,802 iris images captured in the visible spectrum at two locations: indoors (with controlled illumination) and outdoors (with varying environmental scenarios) in two sessions. These images have been captured one at a time using EOS 60D DSLR Canon camera with EFS 60mm f/2.8 Macro USM Fixed lens. In each session of data acquisition, a minimum of six images of each eye when wearing textured contact lens and a minimum of six images of each eye without any lens (real) are acquired. These images are captured indoors and this process is repeated in the outdoor environment. 


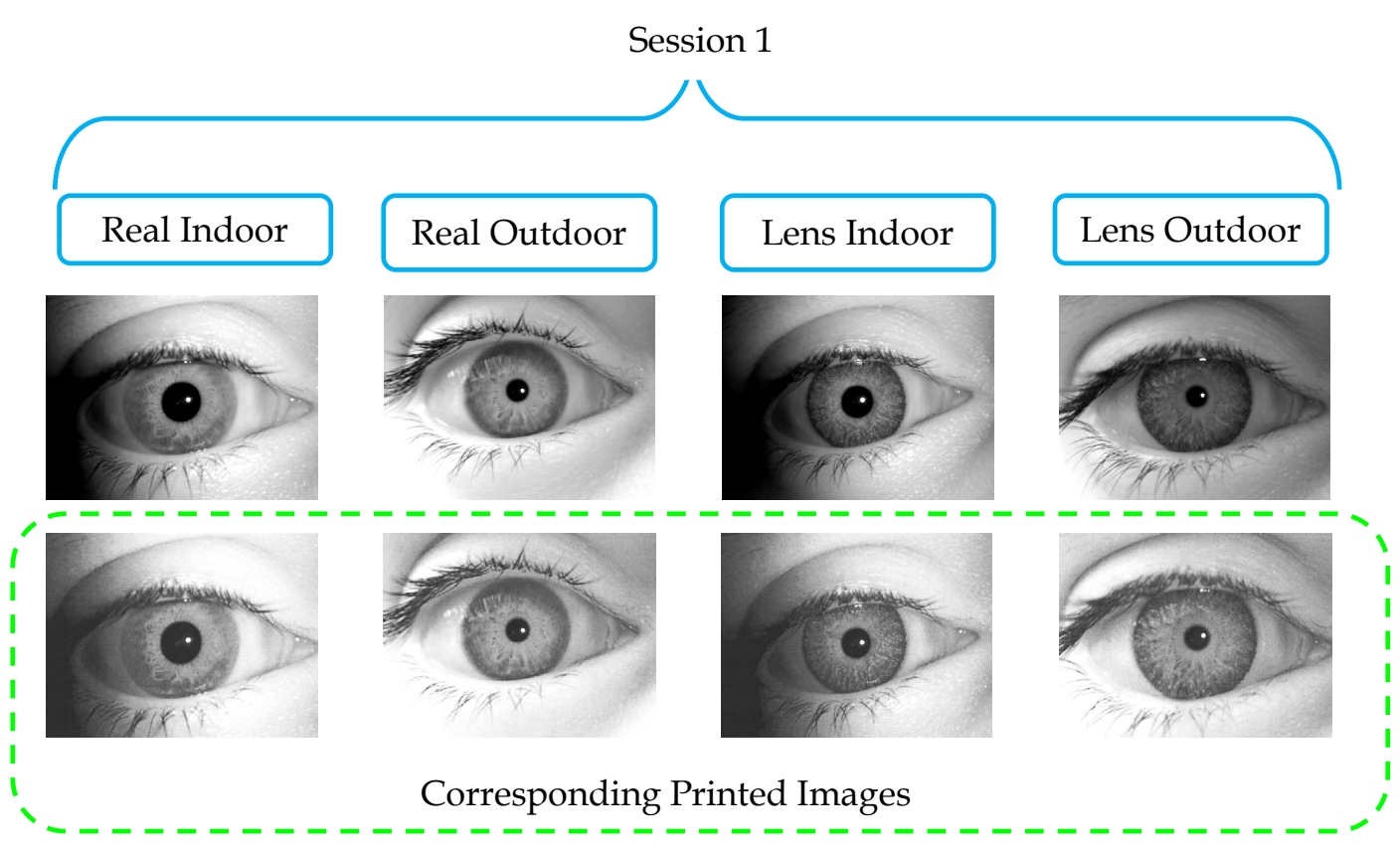

Session 2

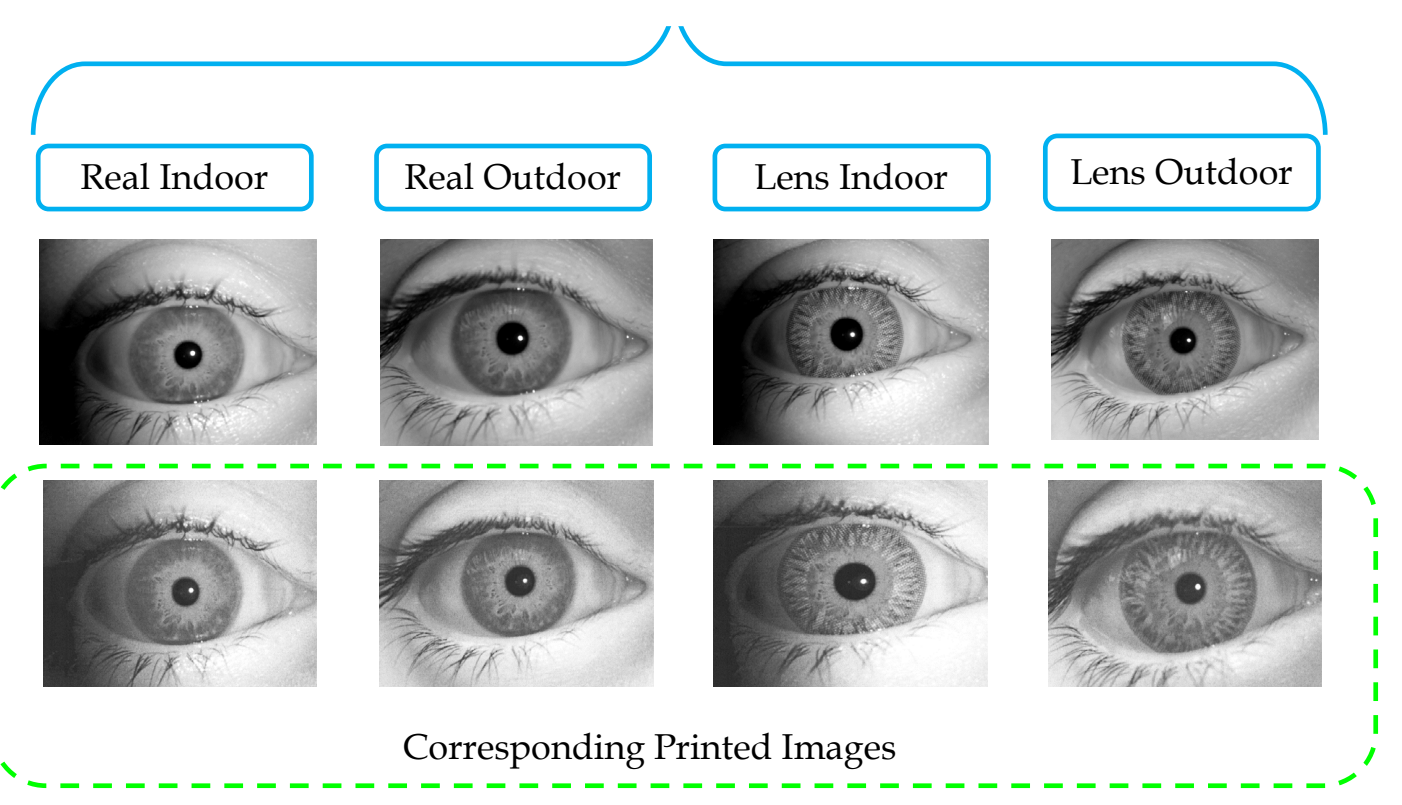

Figure 2.2: Sample iris images of a subject from the proposed Mobile Uncontrolled Iris Presentation Attack Database. 


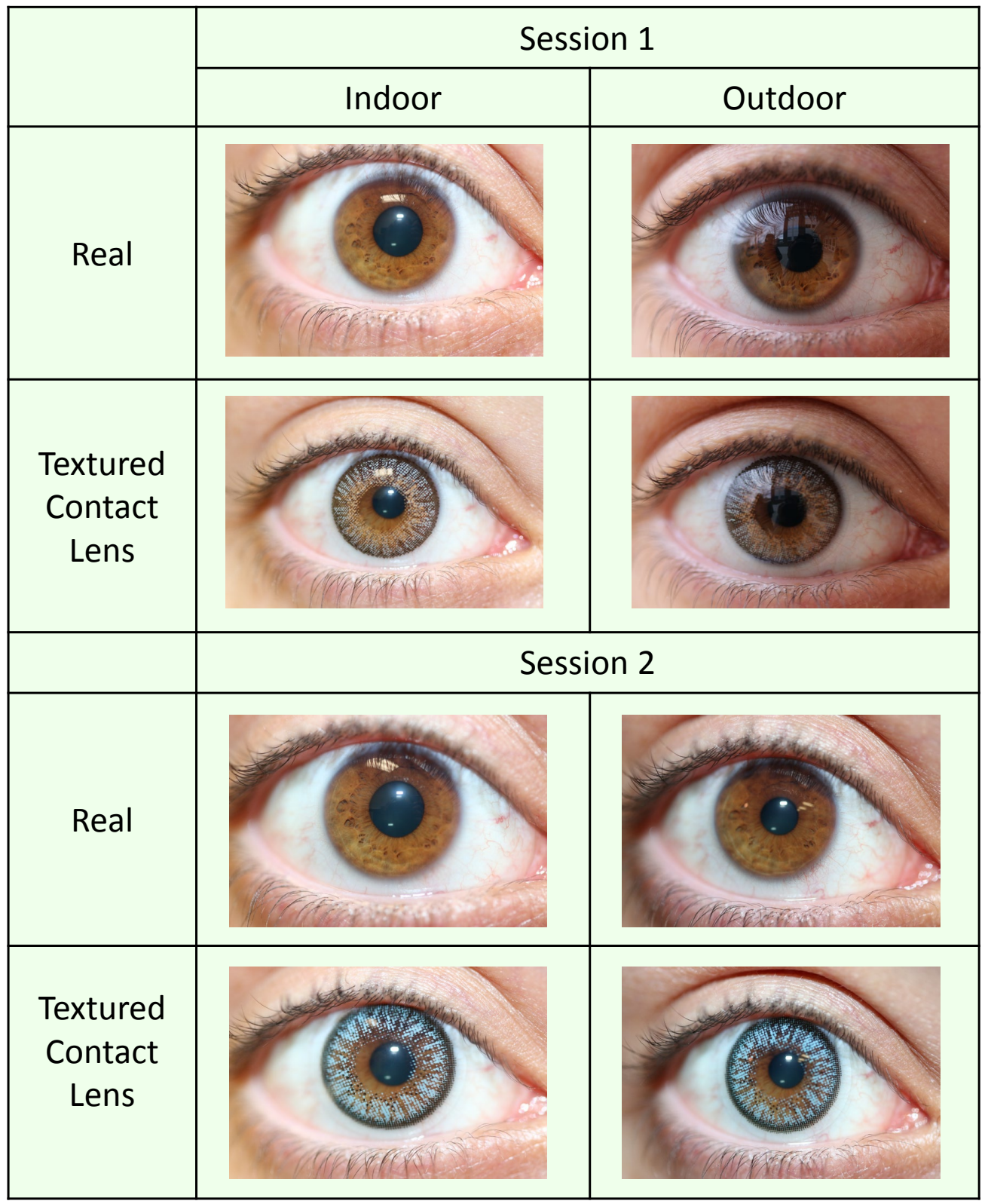

Figure 2.3: Sample images of a subject from the proposed Unconstrained Visible Contact Lens Iris (UVCLI) database demonstrating the variations due to textured contact lens, environmental conditions, and multi-session acquisition. (Best viewed in color) 


\subsection{Unconstrained Visible Spectrum Contact Lens Iris (UVCLI) Database}

Outdoor images have been collected at varying times of the day (such as afternoon and night) and varying weather conditions (such as sunny and cloudy). Majority of the first session outdoor data is collected during the daytime.

The textured contact lenses utilized in this database belong to five different types: CIBA Vision FreshLook Dailies ${ }^{1}$, CIBA Vision Freshlook Colorblends, Bausch \& Lomb Lacelle ${ }^{2}$, Aryan 3-Tone, and Celebration. Varying colors such as pure hazel, turquoise, amethyst, and gray have been selected to create a medley of texture patterns. Also, each participant is provided with a texture contact lens from different manufacturers in different sessions.

In total, the database comprises of iris samples from 35 subjects (70 classes) with 17 females and 18 males between the age of 18-38 years. The database has participants belonging to diverse ethnicities such as Asian, Caucasian, and Hispanic. Table 2.1 summarizes the characteristics of the proposed UVCLI database and Figure 2.3 illustrates sample images of an individual from the database. This new database can be utilized by the research community for visible iris recognition in unconstrained scenarios and textured contact lens detection in the visible spectrum.

\footnotetext{
${ }^{1}$ http://www.freshlookcontacts.com/all-color-contacts/

${ }^{2}$ http://www.bausch.com.my/en/our-products/contact-lenses/cosmetic-lenses/lacelle/
} 


\section{Chapter 3}

\section{Influence of Uncontrolled Environment}

\section{and Textured Contact Lens on Iris}

\section{Recognition}

Environmental variations may significantly impact the quality of the acquired iris image. Factors such as illumination intensity, particularly in daylight condition, can affect the acquisition of iris images. Additionally, the presence of textured contact lenses also negatively impact iris recognition performance. The effect of the textured contact lens on the performance of iris recognition has been studied previously in the literature [10, 21, 40]. However, there is no study evaluating the effect of uncontrolled environment jointly with textured contact lenses on iris recognition systems. Therefore, in this chapter, the efficacy of NIR and visible spectrum based iris recognition systems is evaluated in the presence of two factors: unconstrained environment and textured contact lens using the proposed MUIPAD and UVCLI databases. 


\subsection{Effect of Uncontrolled Environment on NIR Iris Recog- nition}

In this section, the influence of uncontrolled environmental scenario on the performance of NIR iris recognition systems is evaluated. The proposed MUIPAD database is utilized to compare the performance of iris recognition systems on images acquired indoor controlled environment and outdoor uncontrolled environment. For evaluation purposes, two iris recognition systems are utilized: VeriEye ${ }^{1}$, a commercial-off-the-shelf (COTS) system and OSIRIS V4.1 [37], an open-source iris recognition software.

\subsubsection{Experimental Protocol}

To examine the influence of uncontrolled environment on NIR based iris recognition, a gallery set is created comprising one real iris image per class which has been acquired indoors during the first session (70 images in total). Next, the following two probe sets are created for contrasting indoor and outdoor real iris images and the results for Session-I, Session-II, and Combined Session (samples of Session-I and Session-II together) are summarized in Table 3.1:

- Probe-Real-Indoor: Comprises real iris images captured indoors in controlled illumination scenario across the two sessions.

- Probe-Real-Outdoor: Consists of real iris images acquired in the uncontrolled outdoor environment in the two sessions.

\footnotetext{
${ }^{1}$ Www.neurotechnology.com/verieye.html
} 
Table 3.1: Performance (Equal Error Rate \%) of iris recognition systems in the presence of uncontrolled environment and textured contact lens using (a) VeriEye COTS iris recognition system and (b) OSIRIS iris recognition system.

\begin{tabular}{lcc}
\hline & Probe-Real-Indoor & Probe-Real-Outdoor \\
\hline Session-I & 0.58 & 4.95 \\
Session-II & 2.46 & 3.56 \\
Combined Session & 2.63 & 3.29 \\
\hline & Probe-Lens-Indoor & Probe-Lens-Outdoor \\
\hline Session-I & 11.52 & 15.20 \\
Session-II & 12.89 & 13.30 \\
Combined Session & 12.21 & 12.49 \\
\hline
\end{tabular}

(a) VeriEye

\begin{tabular}{lcc}
\hline & Probe-Real-Indoor & Probe-Real-Outdoor \\
\hline Session-I & 5.44 & 17.87 \\
Session-II & 13.62 & 15.45 \\
Combined Session & 12.21 & 15.01 \\
\hline & Probe-Lens-Indoor & Probe-Lens-Outdoor \\
\hline Session-I & 17.27 & 21.58 \\
Session-II & 18.17 & 18.83 \\
Combined Session & 15.74 & 20.55 \\
\hline
\end{tabular}

(b) OSIRIS V4.1 [37]

\subsubsection{Experimental Results}

The equal error rates (EER) values of the two experimental protocols described above are analyzed to compare the effect of environmental conditions on iris recognition. As seen in Table 3.1 and Figure 3.1, it is observed that the Probe-Real-Indoor yields the lower EER value as compared to Probe-Real-Outdoor for both, VeriEye and OSIRIS. This EER value obtained using real to real iris image matching which are acquired indoors (Session-I) serves as the baseline for subsequent analysis. Upon analyzing Probe-Real-Outdoor values, the same- 


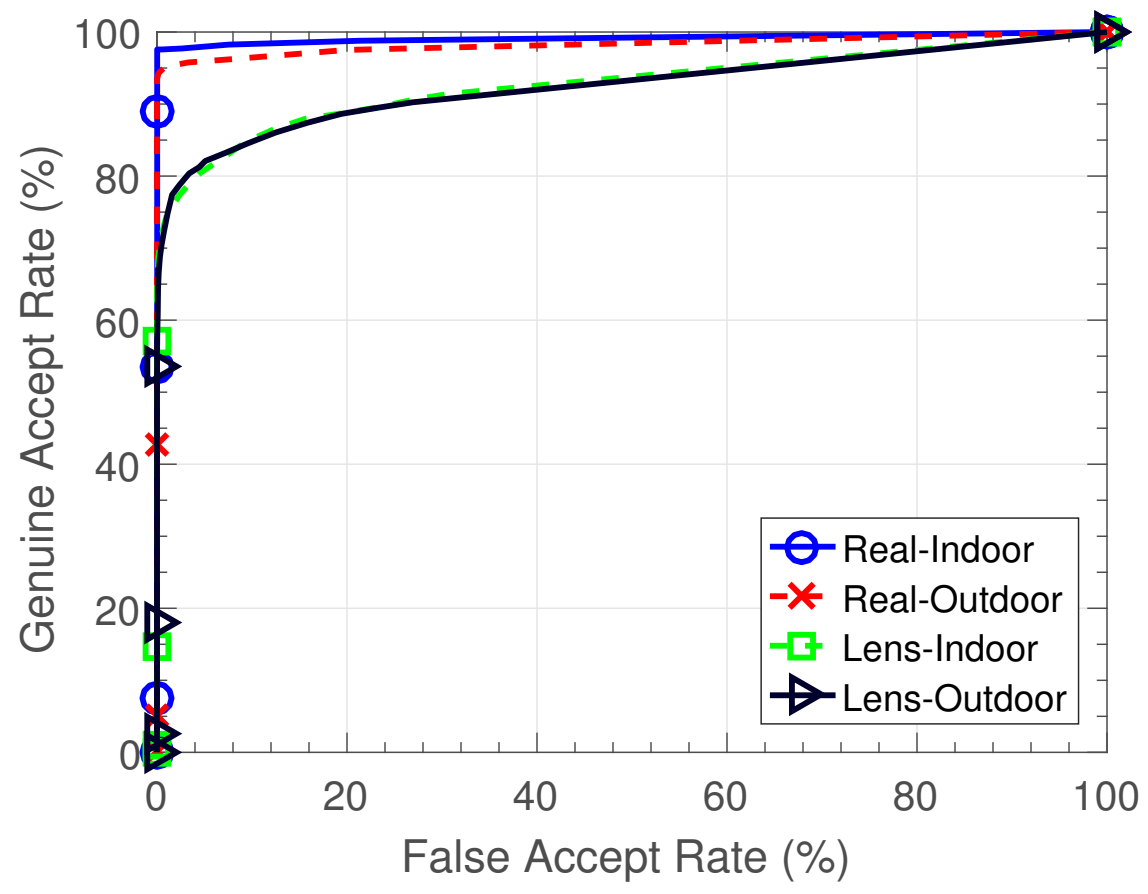

(a) VeriEye

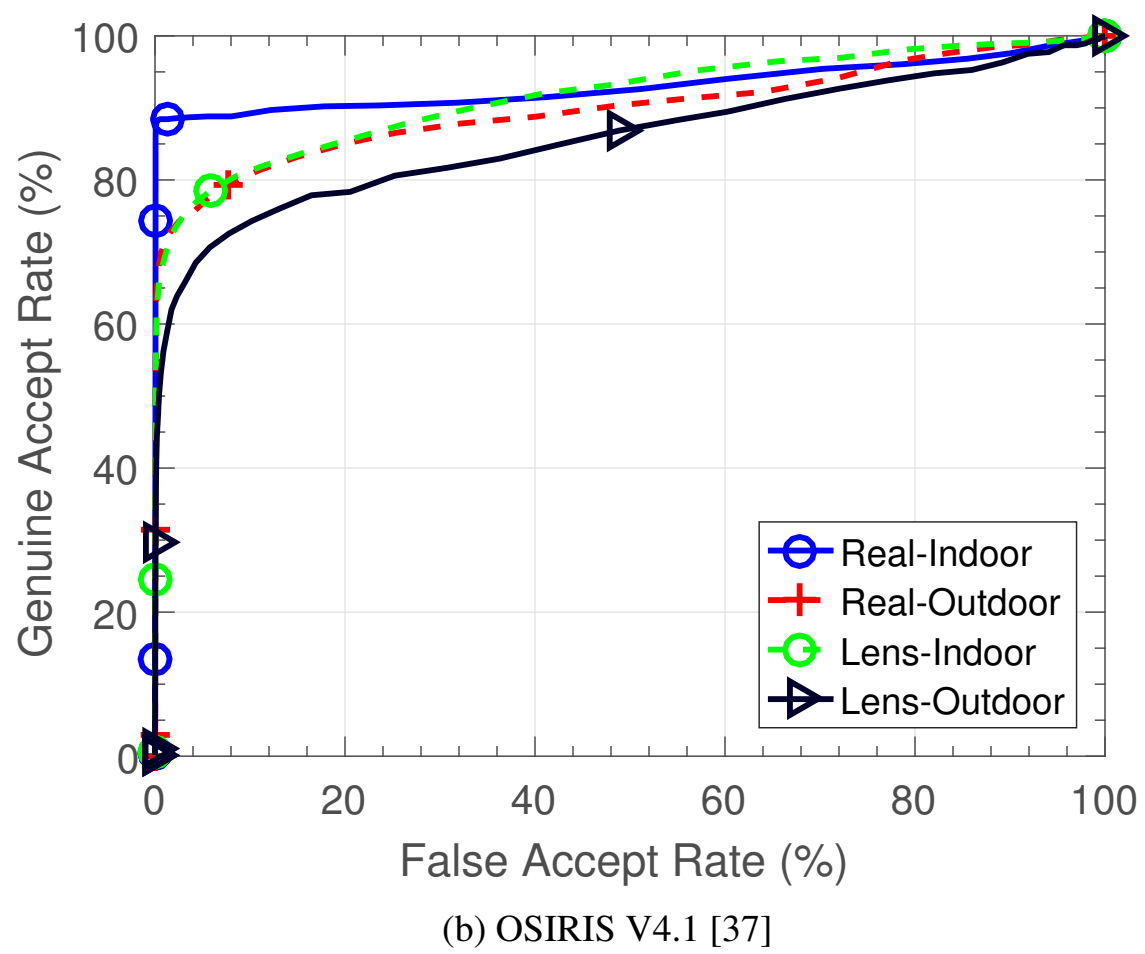

Figure 3.1: ROC curves illustrating the influence of uncontrolled environment and textured contact lens on the performance of iris recognition systems on the combined session. 
session EER increases by $1.10 \%-4.37 \%$ for VeriEye and $1.83 \%-12.43 \%$ for OSIRIS with respect to the baseline. This increase in error rates can be attributed to the challenges added by the uncontrolled environment in images acquired outdoors due to varying illumination and other environmental factors. This highlights the need to develop better iris recognition systems which can operate in both controlled and uncontrolled environmental scenarios.

\subsection{Effect of Textured Contact Lens on NIR Iris Recogni-}

\section{tion}

Next, the effect of textured contact lens on NIR iris recognition algorithms is examined. The artificial patterns on textured lenses can lead to misclassifications. Hence, a thorough examination is necessary to evaluate how it may affect the performance of recognition systems, specifically in the uncontrolled environment.

\subsubsection{Experimental Protocol}

The same gallery set comprising one real iris image per class acquired indoors is chosen for this experiment. Two different probes are considered: (i) Probe-Lens-Indoor constitutes textured lens images captured indoors with controlled environment across the two sessions, and (ii) Probe-Lens-Outdoor comprises textured lens images captured outdoors in the two sessions. The two probe sets are evaluated using VeriEye and OSIRIS and the results are analyzed subsequently. 


\subsubsection{Experimental Results}

As shown in Table 3.1 and Figure 3.1, a consistent decrease in performance for both the iris recognition systems as compared to the baseline is observed when the subjects wear textured contact lens. These textured contact lenses conceal the original iris pattern leading to poor matching performance. For VeriEye system, a decrease of $11 \%$ is observed in the Probe-Lens-Indoor experiment as compared to when the subjects were not wearing any lenses indoors. Similar trends are observed for OSIRIS system with a decrease in performance of $4-12 \%$ in the outdoors and indoors experiment. The results highlight the critical nature of presentation attacks due to textured contact lenses which can be used to easily circumvent iris recognition systems.

\subsection{Effect on Visible Spectrum Iris Recognition}

In the previous section, the impact of textured contact lens and unconstrained environment on traditional NIR iris recognition systems is analyzed. In this section, the first study which analyzes the effect of textured contact lens and unconstrained environmental conditions on the performance of visible spectrum based iris recognition is presented.

\subsubsection{Iris Recognition Algorithms for Evaluation}

The first step in the iris recognition pipeline is the segmentation of the iris region in the input image. For this purpose, three existing algorithms are utilized: OSIRIS v4.1 [37], total variation (TV) based algorithm [44], and IrisSeg [13]. OSIRIS V4.1 [37] is an open-source iris recognition software and has been employed for visible spectrum iris segmentation by Raja et al. [34]. TV-based segmentation algorithm [44] is proposed by Zhao and Kumar for segmenting unconstrained iris images including visible spectrum and they have demonstrated 
superior performance on databases such as UBIRIS.v2 [32] as compared to other approaches. IrisSeg [13] has been recently introduced specifically for segmenting non-ideal irises. Out of these three approaches, the TV-based algorithm yields the best segmentation outputs. Both OSIRIS and IrisSeg are not able to detect the iris-pupil boundary, particularly, in the images that are captured outdoors. It should be noted that various color channels were also explored to enhance segmentation outputs. It is observed that there is a significant decrease in the overall contrast between the pupil and iris region in the images collected in the visible spectrum. This significantly reduces the segmentation performance of the tested algorithms and in many cases, the entire iris is marked as the pupil region. Figure 3.2 demonstrates some sample segmentation outputs and Hence, there is a need to develop better iris segmentation algorithms for unconstrained visible spectrum iris images.

As the goal of this experiment is to compute baseline iris recognition performance to determine the effect of the presence of textured contact lens and environmental variations, feature extraction and matching are performed using the classical iris recognition approach by Masek and Kovesi [27] after segmenting the iris region. This is similar to the approach followed by Zhao and Kumar [44] for visible spectrum iris recognition. Feature extraction is performed by normalizing the segmented iris into rectangular images followed by computation of 1D log-Gabor filters. The templates are created by quantization of these features to four levels and are matched using Hamming distance. For investigating the performance of unconstrained visible spectrum iris recognition, a commercial-off-the-shelf system (VeriEye) is also utilized. However, it failed to segment the iris region in the majority of the images.

\subsubsection{Experimental Protocol}

The proposed UVCLI database has real and textured contact lens images of each subject captured indoors and outdoors across two sessions. An experimental protocol is designed to 


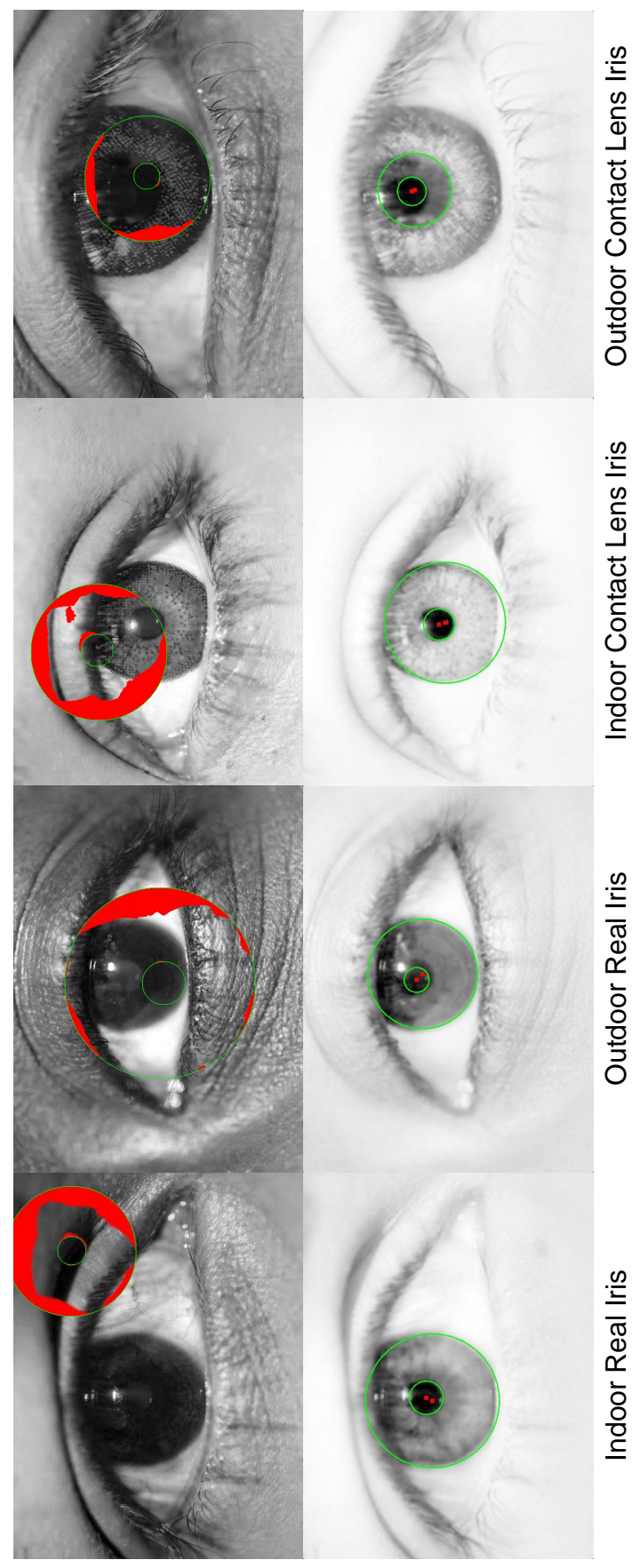

Figure 3.2: Sample segmentation outputs of visible iris images from the proposed UVCLI database using OSIRIS V4.1 [37] (row 1) and TV based segmentation algorithm [44] (row 2). (Best viewed in color) 
Table 3.2: Iris recognition performance (EER\%) on the proposed UVCLI database.

\begin{tabular}{|l|l|c|c|}
\hline Session & Environment & Real & Textured Contact Lens \\
\hline \multirow{2}{*}{ Combined } & Indoor & 19.55 & 38.35 \\
\cline { 2 - 4 } & Outdoor & 37.65 & 42.57 \\
\hline \multirow{2}{*}{ Session 1 } & Indoor & 13.44 & 40.70 \\
\cline { 2 - 4 } & Outdoor & 43.39 & 40.97 \\
\hline \multirow{2}{*}{ Session 2 } & Indoor & 19.97 & 37.76 \\
\cline { 2 - 4 } & Outdoor & 33.55 & 39.70 \\
\hline
\end{tabular}

evaluate the effect of textured contact lenses on the performance of iris recognition system. In this experiment, one real (without contact lens) image per class acquired indoors in the first session are combined to form the gallery. This experiment simulates the scenario where the iris images used for enrollment are acquired in a controlled illumination setting. The remaining visible spectrum iris images are utilized to form the probe set to analyze the impact of textured contact lenses on visible iris recognition.

\subsubsection{Experimental Results}

Table 3.2 and Figure 3.3 show the visible spectrum based iris recognition accuracy on the experimental protocol described above and the results are analyzed subsequently.

As seen in Figure 3.3 and the Combined row of Table 3.2, the best iris recognition performance is observed when the probe contains real images. On further analysis, it is observed that the probe containing images captured indoors in the same session as the gallery (session 1) yields the lowest EER of $13.44 \%$. This result serves as the baseline for the subsequent comparisons as this gallery-probe set pair is expected to have minimum intra-class variations.

On the other hand, when the real gallery images captured indoors are matched with real probe images captured in an outdoor environment, there is a drastic decrease in the iris 


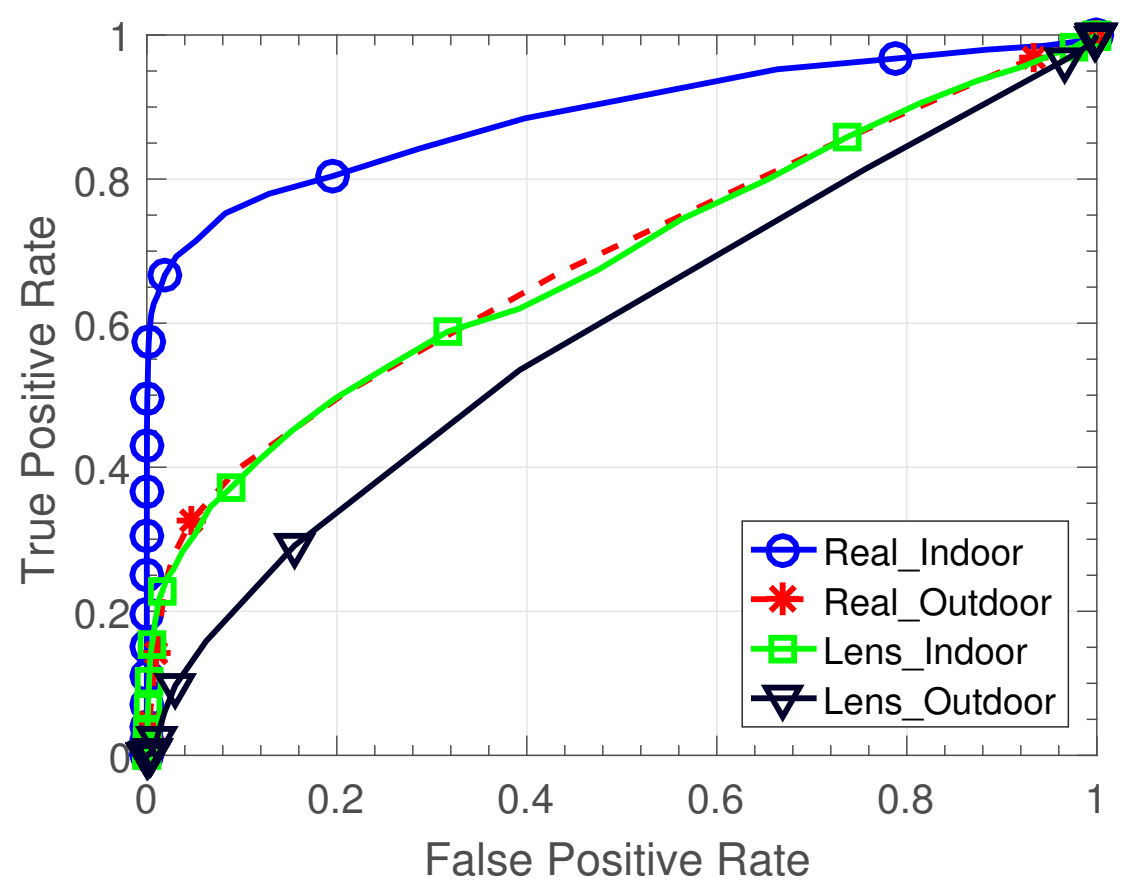

Figure 3.3: ROC curves showing the performance of visible iris recognition on the proposed UVCLI database (Session 1 and Session 2 combined).

recognition performance. It is observed that approximately $30 \%$ increase in EER (session 1 real outdoor) and 20\% increase in EER (session 2 real outdoor) in these cases with respect to the baseline. This highlights the challenging nature of the iris images captured in outdoor environment due to factors such as specular reflection and varying illumination. The $10 \%$ EER increase in session 1 outdoor real images probe as compared to session 2 outdoor real images probe can be attributed to the fact that majority of session 1 outdoor samples are acquired in daylight, which can lead to specular reflections and other illumination issues in the acquired images.

Next, the effect of the presence of textured contact lens in the probe set is analyzed and the EER for the same is shown in the rightmost column of Table 3.2. It is observed that textured contact lenses reduce the performance of visible spectrum iris recognition system as compared to the baseline. The EER of textured contact lenses probe images ranged from 
$37.76 \%$ to $40.97 \%$ EER in different environments and sessions. This result illustrates that textured contact lenses significantly conceal the original iris pattern which in turn, can be utilized by individuals to conceal their identity.

In summary, these experiments highlight that textured contact lens significantly degrades the performance of iris recognition system in the visible spectrum. This can lead to intentional or unintentional concealment of identity. Additionally, it is very challenging to segment visible spectrum iris images acquired in an outdoor environment due to varying illumination conditions and future visible spectrum iris recognition algorithms need to account for these challenges.

\subsection{Textured Contact Lens for Identity Impersonation and Obfuscation}

The previous experiments highlight the scope of improvement in designing algorithms for recognizing iris images in the outdoor environment as well as textured contact lens images. Due to the increasing popularity of textured contact lenses, it is imperative to study if these lenses can be utilized intentionally or unintentionally for hiding own identity or impersonating someone else's identity. However, no study has been undertaken to analyze the security implications of wearing textured contact lens at the time of enrollment. Hence, we present the first quantitative analysis of the efficacy of textured contact lenses on identity impersonation.

\subsubsection{Experimental Protocol}

This experiment is undertaken to understand how current iris recognition systems perform if subjects wear textured contact lenses during the enrollment phase. In this experiment, all the images of subjects wearing textured contact lenses are considered. The scores from 

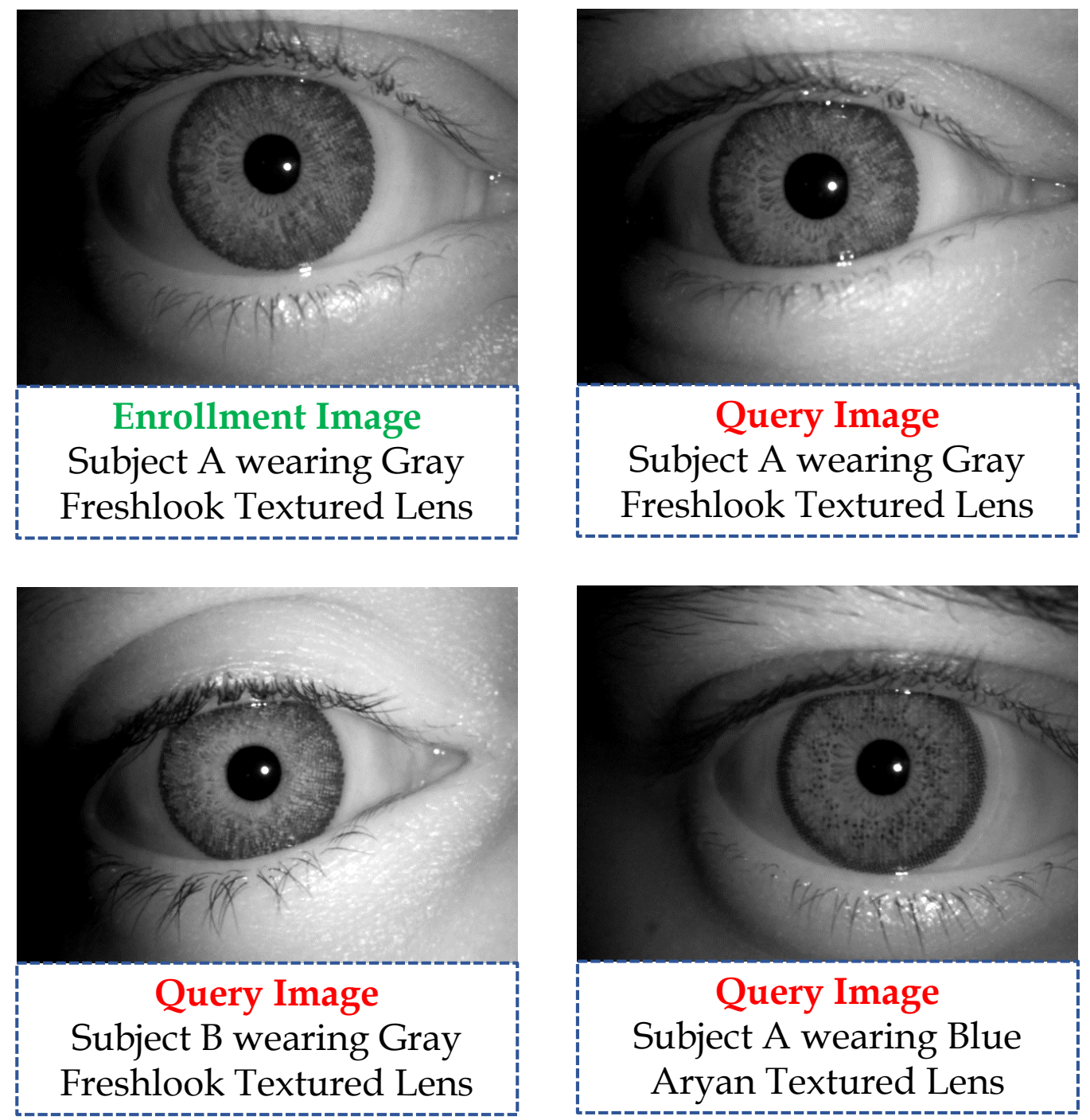

Figure 3.4: Illustrating sample instances of identity impersonation and identity obfuscation using textured contact lenses. 
the pairwise matching of iris images by VeriEye system are computed and the following scenarios are created and shown in Figure 3.4:

- SameSubject-SameLens: Let us assume that subject $A_{1}$ wears textured contact lens $T_{1}$ for enrollment. This experiment replicates the scenario where at the time of the query, the subject $A_{1}$ is wearing the same textured lens $T_{1}$ as the enrollment phase.

- SameSubject-DifferentLens: This depicts the obfuscation scenario where the subject may try to intentionally or unintentionally evade recognition. In this case, subject $A_{1}$ wears a different lens $T_{2}$ than the one worn during the enrollment phase (lens type $T_{1}$ ).

- DifferentSubject-SameLens: This scenario depicts the impersonation scenario where an attacker may utilize textured contact lenses for impersonating a subject enrolled in the system. For instance, subject $A_{2}$ wears the same textured lens $T_{1}$ as subject $A_{1}$ and aims to impersonate as $A_{1}$.

\subsubsection{Experimental Results}

To evaluate the effectiveness of textured contact lenses in obfuscation and impersonation, scores obtained using VeriEye in each of the three scenarios described above are analyzed. To mimic real-world scenarios, the score value at $0.01 \%$ False Accept Rate (FAR) is chosen as the threshold from the Probe-Real-Indoor baseline experiment. This strict threshold is chosen to minimize false positives. All the scores exceeding this threshold are treated as

Table 3.3: Percentage of scores labeled as genuine by VeriEye for different scenarios of identity impersonation and identity obfuscation.

\begin{tabular}{|c|c|c|}
\hline $\begin{array}{c}\text { SameSubject } \\
\text { SameLens }\end{array}$ & $\begin{array}{c}\text { SameSubject } \\
\text { DifferentLens }\end{array}$ & $\begin{array}{c}\text { DifferentSubject } \\
\text { SameLens }\end{array}$ \\
\hline 73.46 & 37.17 & 2.26 \\
\hline
\end{tabular}




\subsection{Textured Contact Lens for Identity Impersonation and Obfuscation}

genuine and the results are shown in Table 3.3. It can be seen in the SameSubject-SameLens scenario, when the subjects wear the same textured contact lenses as the enrollment phase, $73.46 \%$ of the match scores are considered genuine and the remaining $26.54 \%$ scores are labeled as an impostor at $0.01 \%$ FAR. The result highlights that wearing lenses during the enrollment phase may result in a larger number of false positives during the recognition phase. However, the potential of evasion through obfuscation or impersonation may be a strong motivation to wear such textured contact lenses during the enrollment phase.

For the identity impersonation experiment (DifferentSubject-SameLens), two different subjects wear the same textured contact lenses. It is observed that even at a hard threshold of $0.01 \%$ FAR, $2.26 \%$ of the scores (1,292 instances) are considered genuine by the VeriEye matcher. This high number of false positives reveals a critical security concern where an attacker may gain unauthorized access using textured contact lenses. Hence, this experiment illustrates the potential security implications of identity impersonation when an individual wears the same type of textured contact lenses as another subject enrolled in the system.

The analysis of the obfuscation experiment, depicted in SameSubject-DifferentLens scenario, shows that more than $62.83 \%$ of the scores belonging to genuine users are considered as impostors. This result is attributed due to the fact that the subject is wearing a different textured contact lens during the matching phase. Thus, by wearing a different textured contact lens, an attacker may have a high chance to evade/conceal their original identity. 


\section{Chapter 4}

\section{Benchmarking Existing Textured \\ Contact Lens Detection Algorithms}

The previous chapter illustrates that perpetrators may utilize textured contact lenses for identity impersonation as well as obfuscation. It is also demonstrated that uncontrolled environmental variations deteriorate the verification accuracy of iris recognition systems. Hence, it is imperative to evaluate the efficacy of iris presentation attack detection (PAD) algorithms in detecting textured contact lens as well as examine the influence of uncontrolled environmental scenarios. In this chapter, different PAD algorithms for detecting textured contact lenses are evaluated on the proposed MUIPAD and UVCLI databases.

\subsection{Performance on Mobile Uncontrolled Iris Presentation}

\section{Attack Database}

The following iris PAD algorithms are utilized for evaluation purposes: 
- Local Binary Patterns (LBP): LBP features [29] are utilized to encode texture variations. These features have been popularly used by many researchers to discriminate between real and attack images [14]. In the experiments, LBP features with radius $=1$ and neighbors $=8$ are used in conjunction with Support Vector Machine (SVM) [6] as the classifier.

- Weighted Local Binary Patterns (W-LBP): Zhang et al. [43] proposed to encode texture patterns using W-LBP features along with SVM classifier for classifying textured contact lens iris images. The parameter values defined in [43] are utilized for experimental purposes.

- DESIST: This state-of-the-art iris presentation attack framework [22] for detecting multiple presentation attacks, including textured contact lenses and print attack images. It comprises of structural and textural features with neural network classifier for distinguishing attack images from real iris images. The model configuration described in [22] is followed.

- AlexNet: AlexNet [24] is a deep convolutional neural network which was developed for image classification. It consists of five convolutional layers followed by three fully connected layers. In this research, pre-trained AlexNet features are utilized with SVM classifier to encode and classify texture patterns in the presented iris images.

For subsequent iris PAD experiments, five-fold cross-validation is performed on the proposed MUIPAD database such that the subjects in the training and testing partitions are disjoint. 
Table 4.1: Iris presentation attack detection performance on the proposed Mobile Uncontrolled Iris Presentation Attack Database (MUIPAD). The error values are reported in $\%$.

\begin{tabular}{|l|c|c|c|}
\hline Algorithm & Total Error & APCER & BPCER \\
\hline LBP [29] & 13.00 & 15.36 & 1.23 \\
\hline W-LBP [43] & 23.36 & 23.90 & 20.69 \\
\hline DESIST [22] & 16.36 & 18.17 & 7.32 \\
\hline AlexNet [24] & $\mathbf{1 0 . 2 1}$ & $\mathbf{1 1 . 7 9}$ & $\mathbf{2 . 2 8}$ \\
\hline
\end{tabular}

\subsubsection{Benchmarking PAD performance}

For studying the effect of varying environment and textured contact lens on presentation attack, the performance of existing iris PAD algorithms is evaluated on the proposed MUIPAD database. The proposed database is split into training and testing sets using 5-fold cross validation with disjoint subjects and PAD results are reported using the following metrics [17]:

- Total Error: Rate of all misclassified iris images

- Attack Presentation Classification Error Rate (APCER): Rate of misclassified attack iris images

- Bonafide Presentation Classification Error Rate (BPCER): Rate of misclassified real iris images

In this experiment, train and test data contain real and textured lens iris images captured both indoor and outdoor across different sessions. The result of this experiment is summarized in Table 4.1.

It is observed that using features obtained from deep CNN based AlexNet [24] surpasses other existing PAD algorithms and achieves the lowest total error of $10.21 \%$. It outperforms other approaches by at least $2.79 \%$ with respect to the total error metric. It can be inferred that deep learning based features are able to encode discriminatory information for classifying 
Table 4.2: Iris presentation attack detection performance (in \%) of AlexNet [24] on iris images acquired in unseen environment with textured contact lens. In the Benchmark experiment, training and testing partitions contain real and textured lens iris images captured indoors and outdoors across different sessions.

\begin{tabular}{|l|c|c|c|}
\hline Experiment & Total Error & APCER & BPCER \\
\hline Benchmark & 6.88 & 7.30 & 6.44 \\
\hline $\begin{array}{l}\text { Indoor-Train-Outdoor- } \\
\text { Test }\end{array}$ & 25.09 & 4.68 & 45.41 \\
\hline $\begin{array}{l}\text { Outdoor-Train-Indoor- } \\
\text { Test }\end{array}$ & 7.36 & 5.01 & 9.64 \\
\hline
\end{tabular}

real and attacked iris images. Upon further analysis, it is seen that $11.79 \%$ of attacked iris images and $2.28 \%$ of real iris images are misclassified by AlexNet features [24].

As AlexNet [24] based iris PAD algorithm yields the minimum total error rate on the proposed MUIPAD database, it is selected for performing the subsequent analysis. Studies in the literature [15] have demonstrated that print attack images are easier to detect. Therefore, the focus is on distinguishing textured contact lens images from real iris images. For this Real vs. Textured Contact Lens experiment, training and testing partitions with and without textured contact lens iris images are utilized and remove the print attack images. AlexNet feature [24] based algorithm yields 6.88\% total error with APCER of 7.30\% and BPCER of $6.44 \%$ for this experiment. The comparison of APCER values in Table 4.1 and Table 4.2 shows that intra-class variation in the attack class increases by including print attacks as well as textured contact lens images.

\subsubsection{Evaluating the Effect of Unseen Environment}

After benchmarking the performance of iris PAD algorithms on the proposed MUIPAD database, an in-depth analysis of the effect of the unseen environment is performed on the best performing PAD algorithm (AlexNet [24]). In this experiment, the training and testing 
Table 4.3: Iris presentation attack detection performance (in \%) of AlexNet [24] to showcase the effect of unseen textured contact lens manufacturer.

\begin{tabular}{|l|c|c|c|}
\hline Unseen Manufacturer & Total Error & APCER & BPCER \\
\hline Freshlook & 7.88 & 7.88 & 7.89 \\
\hline Colorblends & 3.81 & 5.52 & 2.08 \\
\hline Bausch + Lomb & 18.44 & 34.28 & 2.32 \\
\hline Aryan & 0.00 & 0.00 & 0.00 \\
\hline Celebration & 6.25 & 0.00 & 12.50 \\
\hline
\end{tabular}

partitions created for Real vs. Textured Contact Lens experiment are refined to create the following scenarios. It should be noted that for both the scenarios, the training and testing sets contain disjoint (unseen) subjects.

- Indoor-Train-Outdoor-Test: In this scenario, the training data contains real and attack images acquired in controlled indoor settings while the testing data contains real and attack images acquired in the uncontrolled outdoor environment. This scenario evaluates the performance of iris PAD when the training is performed using controlled images while the testing contains unseen environmental variations.

- Outdoor-Train-Indoor-Test: In this experimental scenario, the training set comprises real and attack images acquired outdoors while the testing set consists of real and attack iris images acquired indoors.

The results of the experimental scenarios are summarized in Indoor-Train-Outdoor-Test and Outdoor-Train-Indoor-Test rows of Table 4.2. Evaluation of the above scenarios reveals that when the iris PAD algorithm is trained using only indoor iris images and is tested on the outdoor iris images, an increase in error rates is witnessed in comparison to when the algorithm is trained using both indoor and outdoor images in Real vs. Textured Contact Lens experiment. A similar trend is observed when AlexNet [24] is trained with images acquired 
outdoors and tested on images acquired indoors. The increase in error upon encountering iris images which are captured in unseen environmental scenarios illustrate the need to incorporate iris images captured in different environmental settings for developing accurate iris PAD models.

\subsubsection{Examining the Impact of Unseen Textured Contact Lens Manu- facturer}

As seen in Figure 3.4, the patterns of the textured contact lenses may vary from manufacturer to manufacturer, even if the color is the same. In this experiment, the efficacy of deep learning based iris presentation attack detection algorithm (AlexNet [24]) is analyzed in detecting textured contact lenses belonging to unknown manufacturers. For this, the textured contact lenses have been categorized into five groups based on the lens manufacturer type: Freshlook, Colorblends, Bausch + Lomb, Aryan, and Celebration. The experimental setup involves performing a five-fold 'leave one manufacturer out' protocol where textured contact lens images belonging to an unseen manufacturer are used in the testing while the training set contains iris images belonging to the remaining manufacturers. It is ensured that subjects in the training and testing partitions are disjoint.

The results of this experiment are compiled in Table 4.3. It is seen that when the testing set contained unseen iris images from Bausch + Lomb manufacturer, AlexNet [24] based iris PAD algorithm yields the highest total error of $18.44 \%$. This can be associated with realistic looking contact lens patterns from this manufacturer, making it more challenging to distinguish from real iris images. This is also evident from the highest APCER of $34.28 \%$ for Bausch + Lomb lenses. On the other hand, AlexNet [24] based iris PAD algorithm yields the lowest total error of $0 \%$ for Aryan textured contact lenses which may be attributed to artificial patterns on the contact lenses of this manufacturer. These results emphasize that 
while designing iris PAD algorithms for textured contact lens detection, there is a need to include lenses from different manufacturers. It also highlights that the textured contact lens detection performance may vary for different manufacturers.

\subsection{Performance on Unconstrained Visible Spectrum Con- tact Lens Iris Database}

As it is crucial to design algorithms for detecting textured contact lens in a given visible spectrum iris image, in this section, the performance of the three existing PAD algorithms is investigated:

- DESIST [22]

- Multiscale BSIF [33]

- Weighted LBP [43]

\subsubsection{Experimental Setup}

For the purpose of iris presentation attack detection, five-fold cross-validation is performed on the proposed UVCLI database such that the subjects in the training and testing partitions are unseen. Two experimental protocols are developed which are explained subsequently:

Unseen Subject Partition: In this experiment, all visible spectrum real and textured contact lens (attack) images of the training partition subjects are used for training the PAD models. Similarly, all the images of the subjects in the testing partitions are utilized for evaluating the PAD performance.

Unseen Subject Partition and Environment: The objective of this experiment is to evaluate the efficacy of iris presentation attack detection algorithms which are trained only with 
Table 4.4: Iris Presentation Attack Detection Performance on the proposed UVCLI database.

(a) Unseen Subject Partition

\begin{tabular}{|l|c|}
\hline Algorithm & Mean PAD Accuracy (\%) \\
\hline DESIST [22] & $\mathbf{8 2 . 8 5} \pm \mathbf{3 . 7 3}$ \\
\hline Weighted LBP [43] & $78.49 \pm 3.15$ \\
\hline Multiscale BSIF [33] & $63.30 \pm 3.31$ \\
\hline
\end{tabular}

(b) Unseen Subject Partition and Environment

\begin{tabular}{|l|c|}
\hline Algorithm & Mean PAD Accuracy (\%) \\
\hline DESIST [22] & $\mathbf{7 4 . 6 0} \pm \mathbf{5 . 3 3}$ \\
\hline Weighted LBP [43] & $73.88 \pm 2.17$ \\
\hline Multiscale BSIF [33] & $56.29 \pm 8.28$ \\
\hline
\end{tabular}

constrained iris images captured indoors. In this experiment, the training set comprises indoor real and attack images captured in the first session, while the testing set comprises first session outdoor, second session indoor, and second session outdoor images of the subjects in the testing partition.

\subsubsection{Experimental Results}

The results of iris presentation attack detection on the proposed UVCLI database are summarized in Table 4.4 and Receiver Operating Characteristic (ROC) curves are presented in Figure 4.1.

\section{Results of Unseen Subject Partition}

Upon analyzing the results of Unseen Subject Partition iris PAD experiment in Table 4.4a and Figure 4.1a, it is observed that DESIST framework [22] outperforms other approaches by at least $4 \%$ in distinguishing between visible spectrum real and attack (textured contact lens) 


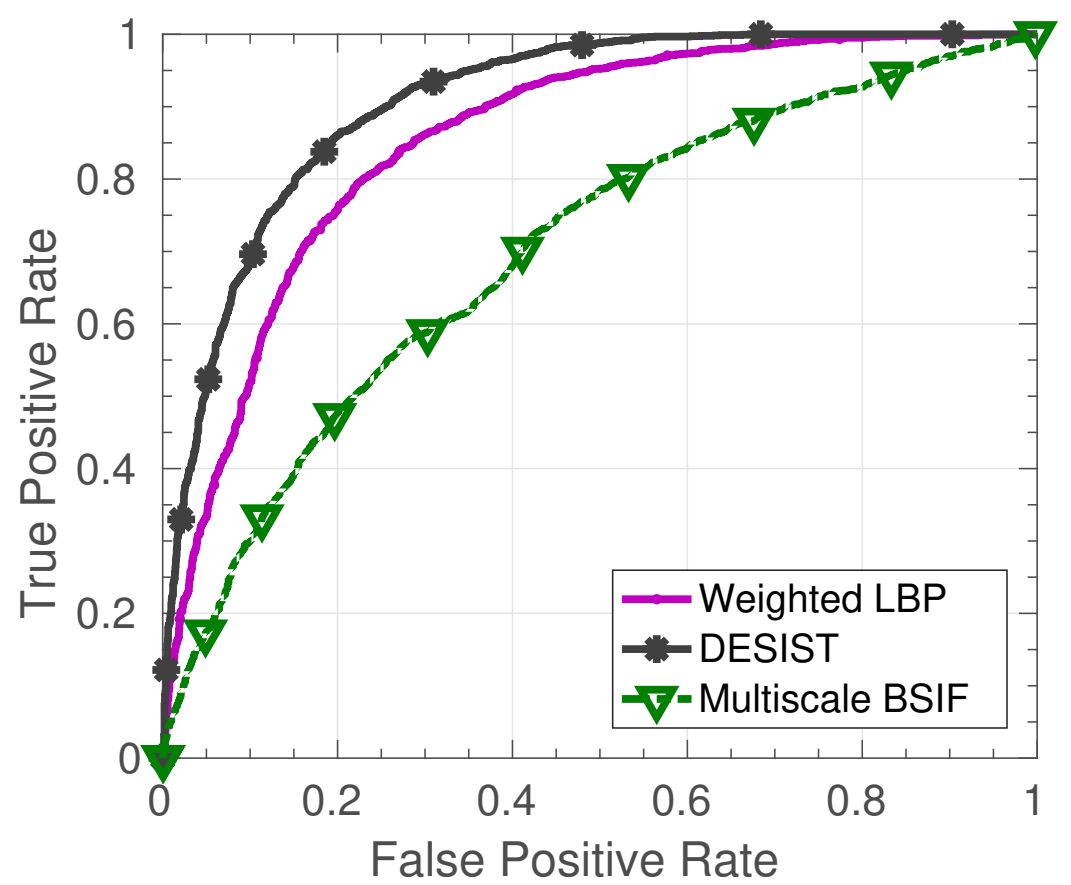

(a) Unseen Subject Partition

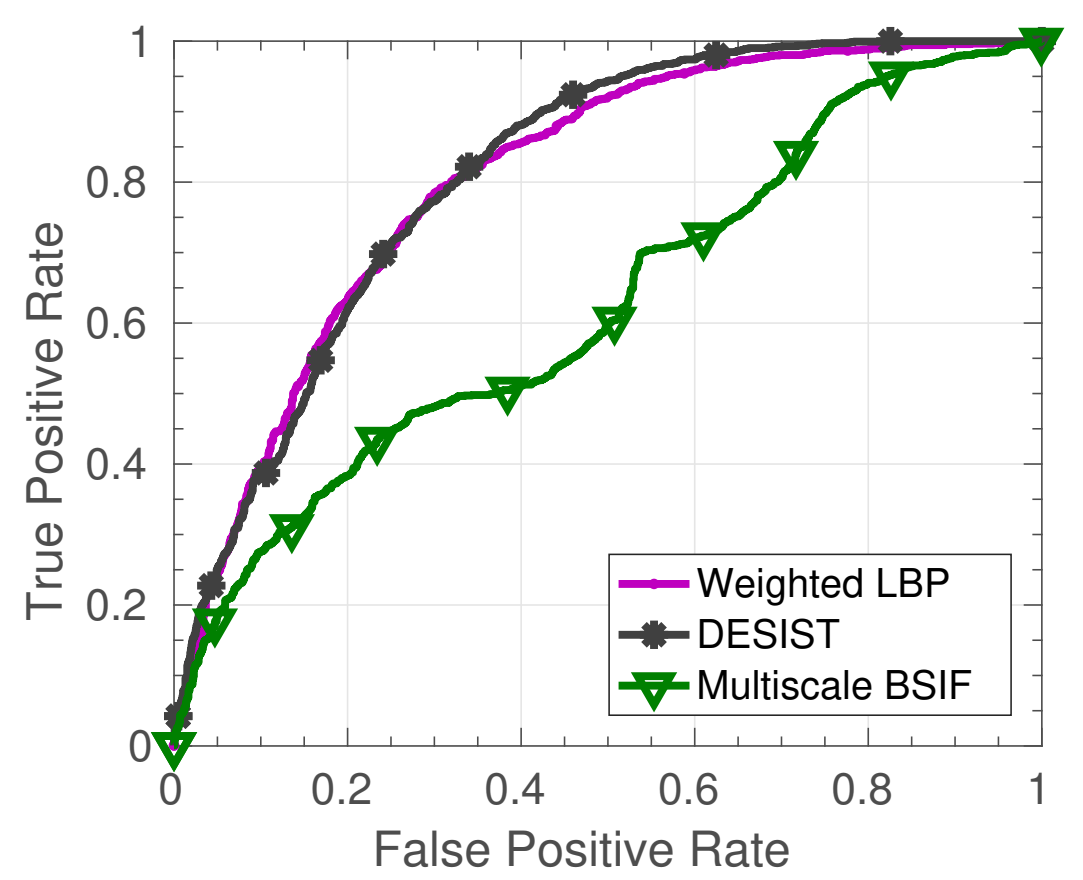

(b) Unseen Subject Partition and Environment

Figure 4.1: ROC curves showing the performance of visible spectrum iris PAD algorithms on the proposed UVCLI database. 
images. The combination of structural and textural features in DESIST is able to encode discriminatory information for iris presentation attack detection.

The performance of iris PAD algorithms are further analyzed with respect to the percentage of real and textured contact lens (attack) images correctly classified in Unseen Subject Partition iris PAD experiment. Using DESIST, $85.00 \%$ of the real images are correctly classified as compared to $79.31 \%$ and $62.07 \%$ by multiscale BSIF and weighted LBP, respectively. On the other hand, $80.79 \%$ of attack images are correctly classified by DESIST. In other words, around $20 \%$ of attack images may be incorrectly classified as real by state-of-the-art iris PAD algorithm.

\section{Results of Unseen Subject Partition and Environment}

For analyzing the effect of environmental variations in visible spectrum iris $\mathrm{PAD}$, the results of Unseen Subject Partition and Environment are evaluated. In this experiment, the training set consists of only real and textured contact lens images captured in an indoor setting with controlled illumination. Table $4.4 \mathrm{~b}$ and Figure $4.1 \mathrm{~b}$ illustrate the results of this experiment. It is observed that the PAD performance of all three algorithms decreases by 5-8\% in Unseen Subject Partition and Environment iris PAD experiment. This can be attributed to the fact that the algorithms are not trained on visible samples that have been acquired in an outdoor environment and did not account for variations such as considerable illumination changes. This showcases that iris PAD algorithms need to be trained with real-world unconstrained iris images.

Additionally, iris PAD analysis of DESIST is performed on session-wise indoor and outdoor visible images. It is observed that second session images which are captured indoors are classified with $82.98 \%$ PAD accuracy as compared to $65.53 \%$ and $73.73 \%$ for first session outdoor and second session outdoor images respectively. The higher classification accuracy 
of second session indoor images can be associated with the fact that the DESIST algorithm is trained with images captured in an indoor environment. Also, it is seen that first session outdoor images are classified with lower accuracy as compared to second session outdoor images. One reason for this can be that the data acquired in the first session outdoor has three times more images collected in sunny weather as compared to the second session. Capturing visible spectrum images in sunny weather can lead to more ambient light and reflection, thus, making it difficult to discern real iris images. 


\section{Chapter 5}

\section{Proposed DenseNet based Algorithm for Iris Presentation Attack Detection}

In the literature of textured contact lens detection, different handcrafted feature based solutions $[9,21,22]$ have been developed. Recently, different deep learning approaches have been successfully used in various supervised and unsupervised machine learning tasks. More specifically, DenseNet based convolutional neural network architectures have shown remarkable performance in several image classification tasks [16]. However, there is no existing work in the literature of iris presentation attack detection or textured contact lens detection which employs this architecture. Motivated by this, the main contributions of this chapter are:

- Proposed a novel framework utilizing DenseNet based deep learning architecture for NIR based iris presentation attack detection.

- Demonstrated results on the Combined Iris database, prepared by combining the images from multiple publicly available databases. It contains more than 270,000 iris 
images with multiple presentation attacks and was created to successfully train a deep learning based model.

- Compared the performance with several state-of-the-art PAD algorithms.

\subsection{Proposed DensePAD Algorithm for Iris Presentation}

\section{Attack Detection}

As shown in Figure 5.1, in DenseNet, each layer is connected to every other layer in a feed-forward fashion as compared to traditional convolutional networks where there is a single connection between each layer and its subsequent layers. For an input image $x$, the $i^{t h}$ layer obtains the concatenation of the feature representations from all the previous layer as input. Thus, the output $x_{i}$ from layer $i$ is computed as:

$$
x_{i}=L_{i}\left(\left[x_{0}, x_{1}, \ldots, x_{i-1}\right]\right)
$$

where $L_{i}$ refers to the non-linear transformations such as batch normalization and pooling. The unique architecture of the DenseNet network strengthens feature propagation, encourages feature reuse, and substantially reduces the number of parameters in the trained model.

In the proposed DenseNet based architecture (DensePAD) for detecting textured contact lens images, iris images of $120 \times 160$ are provided as input. In this architecture, a DenseNet of depth 22 with three densely connected blocks is proposed to classify an input iris image as real or attack (containing textured contact lens). Every dense block is followed by a transition block that consists of a convolution layer and pooling layer to reduce the size of the output. Each dense block in our algorithm consists of six convolution blocks where every convolution block is densely connected. A convolution block itself consists of a convolution 


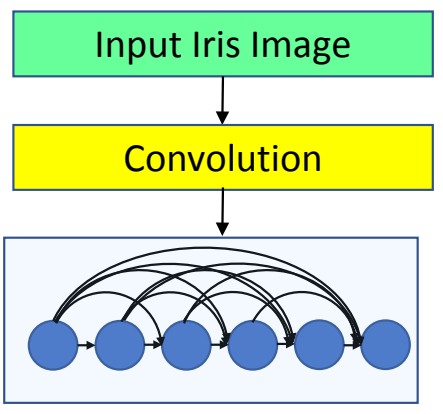

Dense Block 1

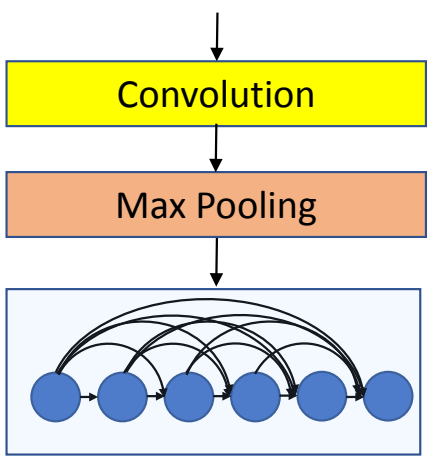

Dense Block 2

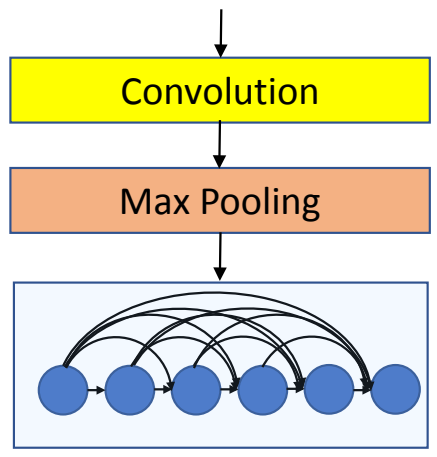

Dense Block 3

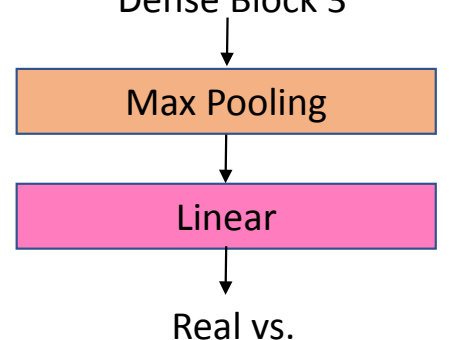

Attack

Figure 5.1: Architecture of proposed DensePAD algorithm for textured contact lens detection. 
Table 5.1: Characteristics of the Combined Iris Database and its constituent databases.

\begin{tabular}{|c|c|c|}
\hline Database & No. of Iris Images & Type(s) of Iris Images \\
\hline $\begin{array}{l}\text { LivDet2013 (Warsaw Subset) } \\
\text { [41] }\end{array}$ & 1,667 & Print and Real \\
\hline $\begin{array}{l}\text { Combined Spoofing Database } \\
\text { [22] }\end{array}$ & 21,525 & $\begin{array}{l}\text { Real, Print, Textured } \\
\text { Contact Lens, and } \\
\text { Synthetic Iris }\end{array}$ \\
\hline NDCLD-2013 [40] & $\begin{array}{l}\text { 4,200 (LG4000) and } \\
900 \text { (AD100) }\end{array}$ & $\begin{array}{l}\text { Real and Textured } \\
\text { Contact Lens }\end{array}$ \\
\hline NDCLD-2015 [8] & 7,300 & $\begin{array}{l}\text { Real and Textured } \\
\text { Contact Lens }\end{array}$ \\
\hline ND-Iris-0405 & 64,980 & Real \\
\hline ND-CrossSensor-Iris-2013 & $\begin{array}{l}117,106(\mathrm{LG} 2200) \& \\
29,845(\mathrm{LG} 4000)\end{array}$ & Real \\
\hline ND-TimeLapseIris-2012 & 6,796 & Real \\
\hline CASIA-Iris-Thousand & 20,000 & Real \\
\hline Combined Iris Database & 274,319 & $\begin{array}{l}\text { Real, Print, Textured } \\
\text { Contact Lens, and } \\
\text { Synthetic Iris }\end{array}$ \\
\hline
\end{tabular}

layer, batch normalization layer, convolution layer and a dropout layer. A growth rate of 12 is used which determines the rate at which the concatenated filters grow.

\subsection{Experimental Evaluation}

\subsubsection{Combined Iris Database}

Various iris presentation attack databases exist in the literature which contain iris images pertaining to a specific presentation attack. However, there is a scarcity of iris databases consisting of multiple presentation attacks. Kohli et al. [22] created Combined Spoofing Dataset (CSD) which was a combination of multiple publicly available spoofing databases. It contained 11,368 attack iris images and 9,325 real iris images from different iris databases. 
Inspired by CSD, the largest iris presentation attack database, Combined Iris database, containing more than 270,000 real and attacked iris images is presented. A key feature of this database is that it comprises iris images acquired from different sensors, subjects of different ethnicities, and multiple presentation attacks. The constituent databases of the Combined Iris database are:

- LivDet2013 (Warsaw Subset) [41] contains 1,667 real and printed iris images.

- Combined Spoofing Database (CSD) [22] comprises various iris presentation attack images such as textured contact lenses, print attack, and synthetic iris images as well as real iris images from different databases $[12,15,25,40]$. The total number of images is 21,525 .

- NDCLD-2013 [40] consists of real and attacked images acquired from two sensors: LG4000 and AD100. 4,200 images have been captured with LG4000 iris sensor while 900 images are acquired with AD100 iris sensor is 900. This database contains iris images with textured contact lens iris images from different manufacturers.

- NDCLD-2015 [8] contains iris images with textured contact lens and real iris images. The total number of images in the database is 7,300 .

The above-mentioned databases contain both, real and attacked iris images. For increasing the real samples in the Combined Iris database, the following databases are also added which contain only real iris images: ND-Iris-0405 [4], ND-CrossSensor-Iris-2013 [5], ND-TimeLapseIris-2012 [2], and CASIA-Iris-Thousand [30]. The characteristics of the Combined Iris database and its constituent databases are summarized in Table 5.1. Figure 5.2 shows sample iris images from different constituent databases of the Combined Iris database along with their image type (real or attack). 

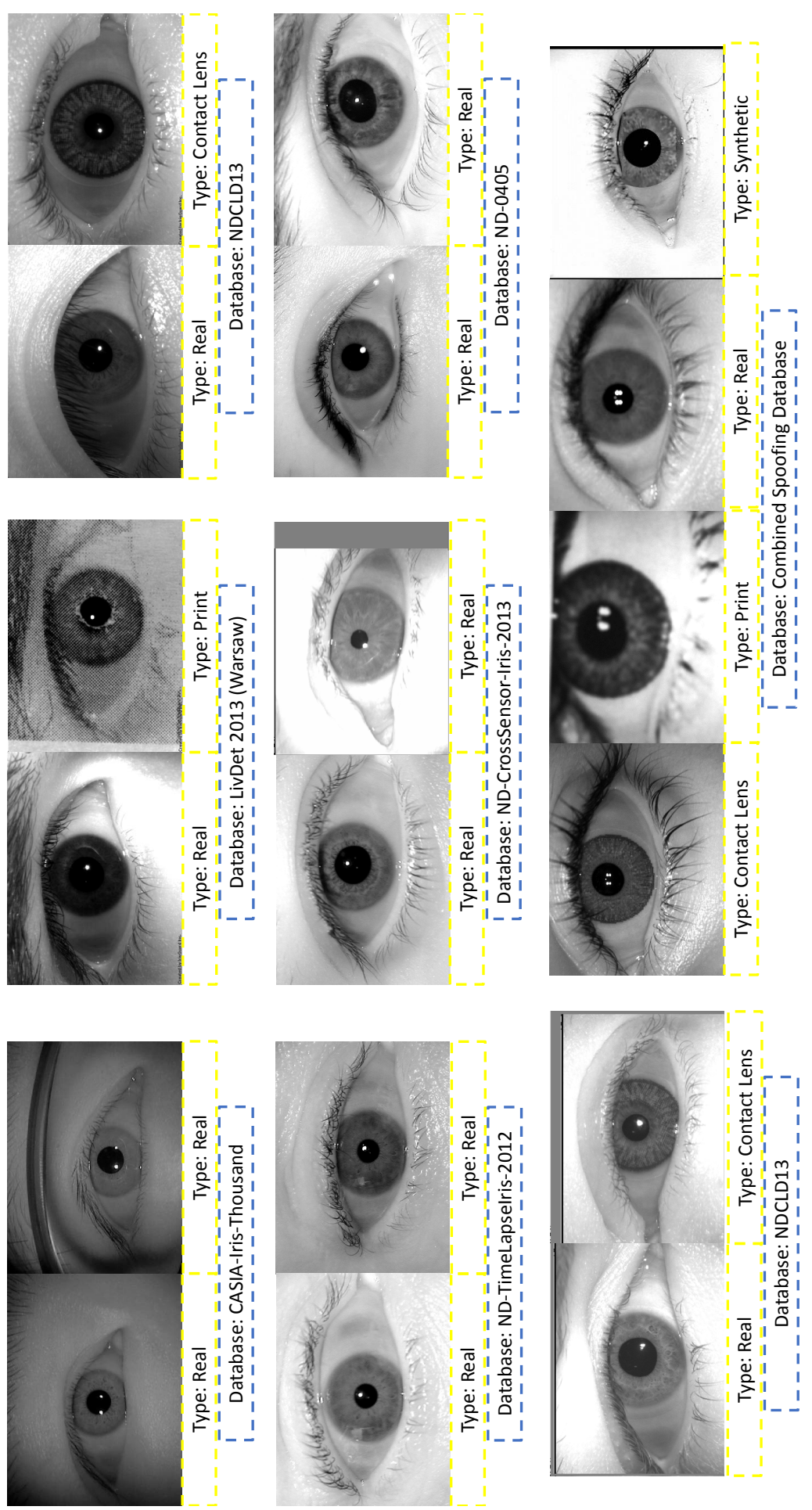

Figure 5.2: Sample iris image types from various constituent databases of the Combined Iris database. 
Table 5.2: The number of real and attacked iris images in each fold for the Combined Iris database.

\begin{tabular}{|c|c|l|c|l|}
\hline \multirow{2}{*}{ Fold } & \multicolumn{2}{|c|}{ Training Partition } & \multicolumn{2}{c|}{ Testing Partition } \\
\cline { 2 - 5 } & Real & Attack & Real & Attack \\
\hline 1 & 210,339 & 9,760 & 51,698 & 2,522 \\
\hline 2 & 208,973 & 9,513 & 53,064 & 2,769 \\
\hline 3 & 209,282 & 9,324 & 52,755 & 2,958 \\
\hline 4 & 209,633 & 9,427 & 52,404 & 2,855 \\
\hline 5 & 211,601 & 9,424 & 50,436 & 2,858 \\
\hline
\end{tabular}

Table 5.3: Iris presentation attack detection performance (\%) of the proposed DensePAD and existing algorithms on the Combined Iris database.

\begin{tabular}{|l|c|c|c|}
\hline Algorithm & Total Error & APCER & BPCER \\
\hline LBP [15] & 22.94 & 80.00 & 20.00 \\
\hline WLBP [43] & 52.75 & 48.18 & 53.00 \\
\hline DESIST [22] & 4.13 & 77.48 & 0.20 \\
\hline Proposed DensePAD & $\mathbf{0 . 9 0}$ & $\mathbf{1 6 . 5 7}$ & $\mathbf{0 . 0 6}$ \\
\hline
\end{tabular}

\subsubsection{Experimental Setup}

The performance of the proposed DensePAD algorithm is evaluated on the Combined Iris database. For experimental evaluation, each constituent database is split into five crossvalidation folds and merged to form the five folds of the Combined Iris database. It is ensured that the subjects in the training and testing partitions are disjoint in every fold. Table 5.2 shows the number of real and attacked iris samples in the training and testing partitions of each fold. Comparative analysis is performed with existing PAD algorithms: LBP [15] , WLBP [43], and DESIST [22].

\subsubsection{Results on Combined Iris Database}

The average total error, APCER, and BPCER values across the five cross-validation folds of the Combined Iris database are summarized in Table 5.3. It is observed that the proposed 
Table 5.4: Iris presentation attack detection performance of the proposed DensePAD algorithm on the proposed Mobile Uncontrolled Iris Presentation Attack Database (MUIPAD). The error values are reported in $\%$.

\begin{tabular}{|l|c|c|c|}
\hline Algorithm & Total Error & APCER & BPCER \\
\hline LBP [29] & 13.00 & 15.36 & 1.23 \\
\hline W-LBP [43] & 23.36 & 23.90 & 20.69 \\
\hline DESIST [22] & 16.36 & 18.17 & 7.32 \\
\hline AlexNet [24] & 10.21 & 11.79 & 2.28 \\
\hline Proposed DensePAD & $\mathbf{9 . 0 6}$ & $\mathbf{1 0 . 1 5}$ & $\mathbf{2 . 1 5}$ \\
\hline
\end{tabular}

DensePAD algorithm yields the best results with a minimum total error of $0.90 \%$. It achieves $16.57 \%$ error in detecting presentation attack iris images and $0.06 \%$ error in detecting real iris images.

Comparative analysis of the proposed DensePAD algorithm is also performed with existing iris PAD algorithms: LBP [15], WLBP [43], and DESIST [22]. It is observed that the DensePAD algorithm outperforms the existing algorithms on the Combined Iris database. It achieves 3-21\% lower total error as compared to LBP, WLBP, and DESIST. With respect to detecting attacked iris images, DensePAD achieves 32-63\% lower APCER as compared to existing algorithms. Similarly, in detecting real iris images, it yields at least $0.14 \%$ lower BPCER as compared to the other algorithms.

\subsubsection{Results on Proposed MUIPAD}

The performance of the proposed DensePAD algorithm is also analyzed on the proposed Mobile Uncontrolled Iris Presentation Attack Database (MUIPAD). For this experiment, MUIPAD is utilized as the testing set while the algorithm is trained on the Combined Iris database. For comparative analysis, the performance of LBP, WLBP, DESIST, and AlexNet is also evaluated and the results are summarized in Table 5.4. 
With respect to the total error, it is observed that the proposed DensePAD algorithm demonstrates improved performance as compared to the other PAD algorithms on MUIPAD. It also achieves the lowest APCER value of $10.15 \%$ and it yields the lowest BPCER value of 2.15\%. These results demonstrate the efficacy of the proposed DensePAD in detecting iris presentation attacks in MUIPAD. 


\section{Chapter 6}

\section{Conclusion and Future Work}

Increasing deployment of smartphones has increased the research in mobile iris devices. Due to their nature, these mobile devices are also utilized in uncontrolled environmental scenarios. At the same time, visible spectrum based iris recognition has emerged into an active area of research. These scenarios have warranted the development of reliable iris recognition algorithms for such uncontrolled environments. Additionally, this growth in iris recognition technology has led to elevated concern regarding its susceptibility towards presentation attacks.

This thesis presented a detailed analysis of the effect of textured contact lenses on iris recognition in the uncontrolled environment. For this, a novel Mobile Uncontrolled Iris Presentation Attack Database (MUIPAD) is presented which consists of more than 10,000 iris images captured in an indoor and outdoor environment using a mobile iris sensor. The database comprises iris images belonging to subjects wearing textured contact lens and without wearing contact lenses (real) along with corresponding print attack images. In this thesis, a detailed analysis is performed to investigate the impact of textured contact lenses on identity impersonation. The results demonstrate that a perpetrator can impersonate 
the identity of an enrolled subject by wearing the same textured contact lens. This result highlights a key security implication of textured contact lenses.

In this thesis, the impact of textured contact lenses in an uncontrolled environment in the visible spectrum is also studied. However, very few databases exist for iris recognition in visible spectrum which are acquired in an unconstrained manner. At the same time, similar to the NIR spectrum, iris recognition in the visible spectrum is also vulnerable to different types of presentation attacks. These experimental results demonstrate that there is a need to actively investigate and mitigate the effect of presentation attacks in iris recognition for the visible spectrum. To promote the research in the area of presentation attack detection of textured contact lenses, the first contact lens database in the visible spectrum is introduced, named as UVCLI database. The database contains over 3,800 real and textured contact lens images belonging to 70 iris classes. This database is acquired with varying unconstrained environmental scenarios and is multi-session in nature. Due to these variations, the database can also be utilized for research in iris recognition in the unconstrained visible spectrum.

Using the UVCLI database, the first detailed analysis of the effect of textured contact lenses on iris recognition in the visible spectrum is presented. It is observed that textured contact lenses degrade the unconstrained iris recognition performance by over $25 \%$ and thus, may be utilized intentionally or unintentionally to attack existing iris recognition systems. To analyze the efficacy of presentation attack detection algorithms on the UVCLI database, three algorithms are utilized. The experimental evaluation highlights the need to develop efficient algorithms for detecting contact lenses in the visible spectrum.

This thesis also presents a novel deep learning based algorithm, DensePAD, for detecting real and attack iris images. The proposed DensePAD algorithm utilizes DenseNet based convolutional neural network architecture to encode discriminatory features for real and attack iris images. Its experimental evaluation on the proposed MUIPAD and combined iris 
database, containing more than 270,000 iris images, showcases its superior performance in classifying real and attack iris images. 


\section{Bibliography}

[1] Statistics and facts about smartphones. https://www.statista.com/statistics/330695/ number-of-smartphone-users-worldwide/. [Online; accessed 16-December-2018].

[2] S. E. Baker, K. W. Bowyer, P. J. Flynn, and P. J. Phillips. Template aging in iris biometrics. In Handbook of Iris Recognition, pages 205-218. Springer, 2013.

[3] S. E. Baker, A. Hentz, K. W. Bowyer, and P. J. Flynn. Degradation of iris recognition performance due to non-cosmetic prescription contact lenses. Computer Vision and Image Understanding, 114(9):1030-1044, 2010.

[4] K. W. Bowyer and P. J. Flynn. The ND-IRIS-0405 iris image dataset. arXiv preprint arXiv:1606.04853, 2016.

[5] R. Connaughton, A. Sgroi, K. W. Bowyer, and P. Flynn. A cross-sensor evaluation of three commercial iris cameras for iris biometrics. In IEEE Computer Vision and Pattern Recognition Workshops, pages 90-97, 2011.

[6] C. Cortes and V. Vapnik. Support vector machine. Machine Learning, 20(3):273-297, 1995.

[7] J. Daugman. How iris recognition works. In The essential guide to image processing, pages 715-739. Elsevier, 2009.

[8] J. S. Doyle and K. W. Bowyer. Robust detection of textured contact lenses in iris recognition using BSIF. IEEE Access, 3:1672-1683, 2015. 
[9] J. S. Doyle, K. W. Bowyer, and P. J. Flynn. Variation in accuracy of textured contact lens detection based on sensor and lens pattern. In IEEE International Conference on Biometrics: Theory, Applications and Systems, pages 1-7, 2013.

[10] J. S. Doyle, P. J. Flynn, and K. W. Bowyer. Automated classification of contact lens type in iris images. In IEEE International Conference on Biometrics, pages 1-6, 2013.

[11] J. Galbally, J. Ortiz-Lopez, J. Fierrez, and J. Ortega-Garcia. Iris liveness detection based on quality related features. In IEEE International Conference on Biometrics, pages 271-276, 2012.

[12] J. Galbally, A. Ross, M. Gomez-Barrero, J. Fierrez, and J. Ortega-Garcia. Iris image reconstruction from binary templates: An efficient probabilistic approach based on genetic algorithms. Computer Vision and Image Understanding, 117(10):1512-1525, 2013.

[13] A. Gangwar, A. Joshi, A. Singh, F. Alonso-Fernandez, and J. Bigun. Irisseg: A fast and robust iris segmentation framework for non-ideal iris images. In IEEE International Conference on Biometrics, pages 1-8, 2016.

[14] D. Gragnaniello, C. Sansone, and L. Verdoliva. Iris liveness detection for mobile devices based on local descriptors. Pattern Recognition Letters, 57:81-87, 2015.

[15] P. Gupta, S. Behera, M. Vatsa, and R. Singh. On iris spoofing using print attack. In IEEE International Conference on Pattern Recognition, pages 1681-1686, 2014.

[16] G. Huang, Z. Liu, K. Q. Weinberger, and L. van der Maaten. Densely connected convolutional networks. In IEEE Conference on Computer Vision and Pattern Recognition, pages 4700-4708, 2017.

[17] Information technology - Biometric presentation attack detection. Standard ISO/IEC 30107-1:2016 - Part 1 - Framework. 
[18] A. Jain, P. Mittal, G. Goswami, M. Vatsa, and R. Singh. Person identification at a distance via ocular biometrics. In IEEE International Conference on Identity, Security and Behavior Analysis, pages 1-6, 2015.

[19] A. K. Jain, A. Ross, and S. Prabhakar. An introduction to biometric recognition. IEEE Transaction on Circuits and Systems for Video Technology, 14(1):4-20, 2004.

[20] R. R. Jillela and A. Ross. Segmenting iris images in the visible spectrum with applications in mobile biometrics. Pattern Recognition Letters, 57:4 - 16, 2015. Mobile Iris CHallenge Evaluation part I (MICHE I).

[21] N. Kohli, D. Yadav, M. Vatsa, and R. Singh. Revisiting iris recognition with color cosmetic contact lenses. In IEEE International Conference on Biometrics, pages 1-7, 2013.

[22] N. Kohli, D. Yadav, M. Vatsa, R. Singh, and A. Noore. Detecting medley of iris spoofing attacks using DESIST. In IEEE International Conference on Biometrics Theory, Applications and Systems, pages 1-6, 2016.

[23] J. Komulainen, A. Hadid, and M. Pietikäinen. Generalized textured contact lens detection by extracting BSIF description from cartesian iris images. In IEEE International Joint Conference on Biometrics, pages 1-7, 2014.

[24] A. Krizhevsky, I. Sutskever, and G. E. Hinton. Imagenet classification with deep convolutional neural networks. In Advances in Neural Information Processing Systems, pages 1097-1105, 2012.

[25] A. Kumar and A. Passi. Comparison and combination of iris matchers for reliable personal authentication. Pattern Recognition, 43(3):1016-1026, 2010.

[26] M. D. Marsico, M. Nappi, D. Riccio, and H. Wechsler. Mobile iris challenge evaluation (MICHE)-I, biometric iris dataset and protocols. Pattern Recognition Letters, 57:17 23, 2015. 
[27] L. Masek and P. Kovesi. Matlab source code for a biometric identification system based on iris patterns. The School of Computer Science and Software Engineering, The University of Western Australia, 2(4), 2003.

[28] I. Nigam, M. Vatsa, and R. Singh. Ocular biometrics: A survey of modalities and fusion approaches. Information Fusion, 26:1 - 35, 2015.

[29] T. Ojala, M. Pietikainen, and T. Maenpaa. Multiresolution gray-scale and rotation invariant texture classification with local binary patterns. IEEE Transactions on Pattern Analysis and Machine Intelligence, 24(7):971-987, 2002.

[30] [Online]. CASIA Iris image database. http://biometrics.idealtest.org.

[31] A. Perala. Princeton identity tech powers galaxy s8 iris scanning. https://mobileidworld. com/princeton-identity-galaxy-s8-iris-003312, 2017. [Online; accessed 16-December2018].

[32] H. Proenca, S. Filipe, R. Santos, J. Oliveira, and L. A. Alexandre. The UBIRIS.v2: A database of visible wavelength iris images captured on-the-move and at-a-distance. IEEE Transactions on Pattern Analysis and Machine Intelligence, 32(8):1529-1535, 2010.

[33] R. Raghavendra and C. Busch. Robust scheme for iris presentation attack detection using multiscale binarized statistical image features. IEEE Transactions on Information Forensics and Security, 10(4):703-715, 2015.

[34] K. B. Raja, R. Raghavendra, and C. Busch. Video presentation attack detection in visible spectrum iris recognition using magnified phase information. IEEE Transactions on Information Forensics and Security, 10(10):2048-2056, 2015.

[35] K. B. Raja, R. Raghavendra, V. K. Vemuri, and C. Busch. Smartphone based visible iris recognition using deep sparse filtering. Pattern Recognition Letters, 57(C):33-42, 2015. 
[36] S. Shah and A. Ross. Generating synthetic irises by feature agglomeration. In IEEE International Conference on Image Processing, pages 317-320, 2006.

[37] G. Sutra, B. Dorizzi, S. Garcia-Salicetti, and N. Othman. A biometric reference system for iris, OSIRIS, version 4.12012. Technical report, 2013.

[38] C. W. Tan and A. Kumar. Accurate iris recognition at a distance using stabilized iris encoding and Zernike moments phase features. IEEE Transactions on Image Processing, 23(9):3962-3974, 2014.

[39] M. Trokielewicz. Iris recognition with a database of iris images obtained in visible light using smartphone camera. In IEEE International Conference on Identity, Security and Behavior Analysis, pages 1-6, 2016.

[40] D. Yadav, N. Kohli, J. S. Doyle, R. Singh, M. Vatsa, and K. W. Bowyer. Unraveling the effect of textured contact lenses on iris recognition. IEEE Transactions on Information Forensics and Security, 9(5):851-862, 2014.

[41] D. Yambay, J. S. Doyle, K. W. Bowyer, A. Czajka, and S. Schuckers. Livdet-iris 2013 iris liveness detection competition 2013. In IEEE International Joint Conference on Biometrics, pages 1-8, 2014.

[42] D. Yambay, B. Walczak, S. Schuckers, and A. Czajka. Livdet-iris 2015 - iris liveness detection competition 2015. In IEEE International Conference on Identity, Security and Behavior Analysis, pages 1-6, 2017.

[43] H. Zhang, Z. Sun, and T. Tan. Contact lens detection based on weighted LBP. In IEEE International Conference on Pattern Recognition, pages 4279-4282, 2010.

[44] Z. Zhao and A. Kumar. An accurate iris segmentation framework under relaxed imaging constraints using total variation model. In IEEE International Conference on Computer Vision, pages 3828-3836, 2015. 